A MACHINE PROGRAM FOR CALCULATING SLOW NEUTRON SCATTERING CROSS SECTIONS WITH APPLICATION TO WATER
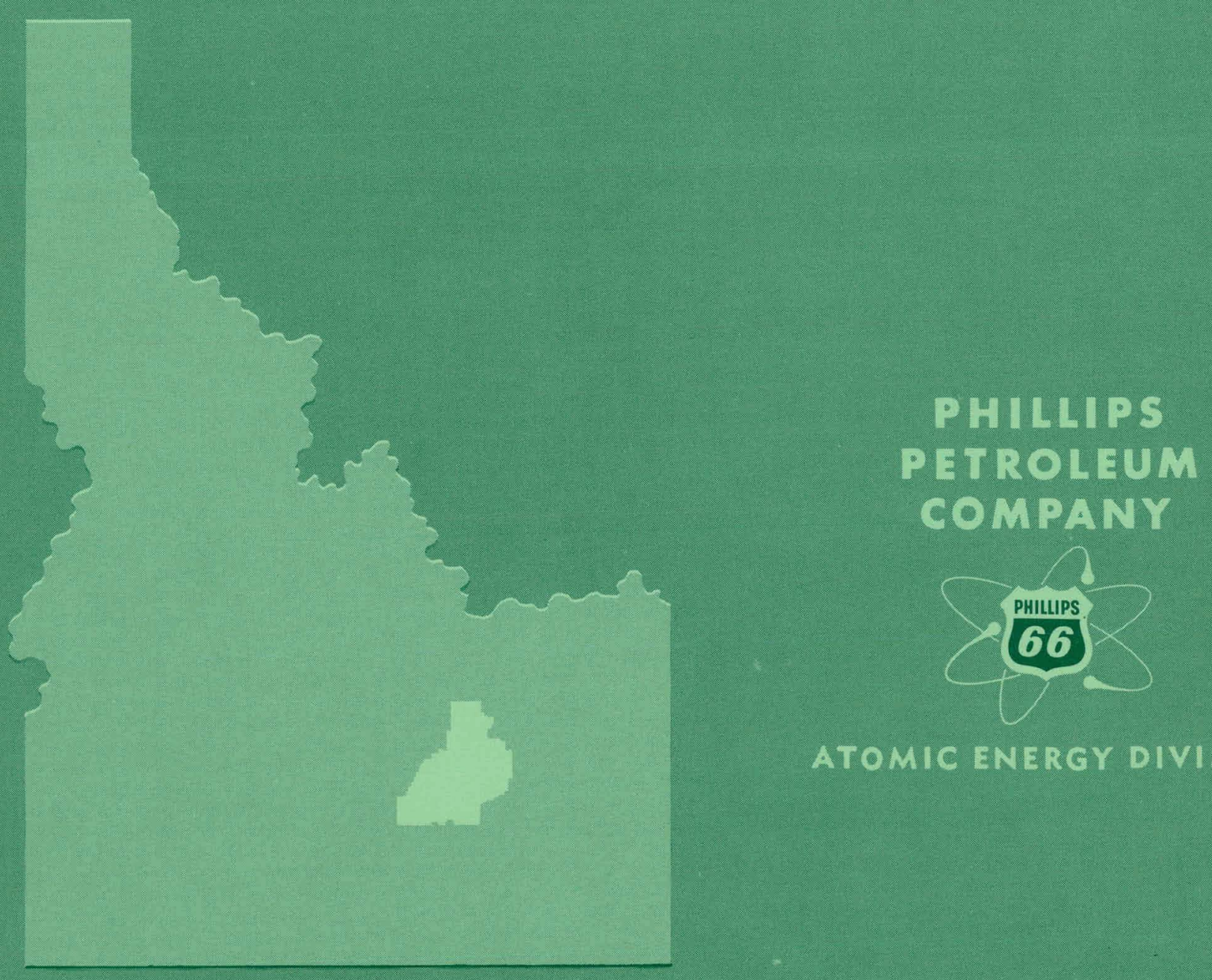

ATOMIC ENERGY DIVISION

NATIONAL REACTOR TESTINE STATION US ATOMIC ENERCY COMMISSION 


\section{DISCLAIMER}

This report was prepared as an account of work sponsored by an agency of the United States Government. Neither the United States Government nor any agency Thereof, nor any of their employees, makes any warranty, express or implied, or assumes any legal liability or responsibility for the accuracy, completeness, or usefulness of any information, apparatus, product, or process disclosed, or represents that its use would not infringe privately owned rights. Reference herein to any specific commercial product, process, or service by trade name, trademark, manufacturer, or otherwise does not necessarily constitute or imply its endorsement, recommendation, or favoring by the United States Government or any agency thereof. The views and opinions of authors expressed herein do not necessarily state or reflect those of the United States Government or any agency thereof. 


\section{DISCLAIMER}

Portions of this document may be illegible in electronic image products. Images are produced from the best available original document. 
PRICE $\$ 1.25$

AVAILABLE FROM THE

OFFICE OF TECHNICAL SERVICES

U. S. DEPARTMENT OF COMMERCE

WASHINGTON 25 , D. C.

\section{LEGAL NOTICE}

This report was prepared as an account of Government sponsored work. Neither the United States, nor the Commission, nor any person acting on behalf of the Commission:

A. Makes any warranty or representation, express or implied, with respect to the accuracy, completeness, or usefulness of the information contained in this report, or that the use of any information, apparatus, method, or process disclosed in this report may not infringe privately owned rights; or

B. Assumes any lialnilities with respect to the nse of, or for damages resulting from the use of any information, apparatus, method, or process disclosed in this report.

As used in the above, "person acting on behalf of the Commission" includes any employee or contractor of the Commission, or employee of such contractor, to the extent that such employee or contractor of the Commission, or employee of such contractor prepares, disseminates, or provides access to, any information pursuant to his employment or contract with the Commission, $\mathrm{nr}$ his employment with such contractor. 


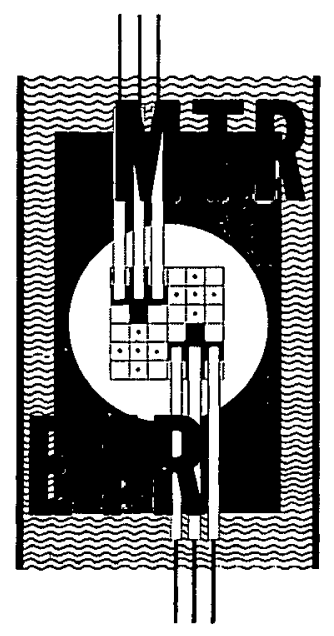

IDO-16984

AEC Research and Development Report

Physics

TID-4500 ( 30 th Ed.)

Issued: July 1964

A MACHINE PROGRAM FOR CALCULATING

SLOW NEUTRON SCATTERTNG CROSS SECTIONS WITH APPLICATION TO WATER

H. L. McMurry

L. J. Gannon

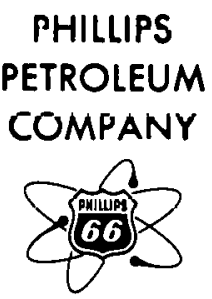

Atomic Energy Division

Contrat AT $(10.1) .205$

Idaho Operations Office

U. S. ATOMIC ENERGY COMMISSION 
THIS PAGE

\section{WAS INTENTIONALLY LEFT BLANK}




\title{
A MACHINE PROGRAM FOR CALCULATING \\ SLOW NEUTRON SCATTERING CROSS SECTIONS WITH APPLICATION TO WATER
}

\author{
H. I. McMurry and L. J. Gannon
}

\section{ABSTRACT}

The scattering by an atom in a complex molecular aggregate is calculated from an equation involving factors associated with the over-all translational and rotational degrees of freedom of the aggregate, together with its internal vibrations.

The rotational and translational factors are quite easy to calculate. The vibrational factor appears as an infinite sum of terms associated with all possible mechanisms of excitation and de-excitation of the normal modes. For practical purposes it is necessary to limit the number of modes which contribute terms to the vibrational factor by treating modes classically whenever the energy transfer in a collision exceeds the quantum spacing of a vibrational level sufficiently. Furthermore, computations must be restricted to only the significant terms in the vibrational factor.

The report sets out criteria for treating modes classically, and for selecting only the significant terms in the vibrational factor. A program for making the calculations on the IBM 7040 is described.

To illustrate the method and the program, calculations are made for water using the Nelkin model and two elaborations of the Nelkin model which allow for more internal vibrational degrees of freedom. The added complexity yields much better agreement between observed and calculated partial differential scattering cross section data for water. 
THIS PAGE

\section{WAS INTENTIONALLY LEFT BLANK}


CONTENTS

Abstract . . . . . . . . . . . . . . . . ii

I Introduction. . . . . . . . .......... I

II The Scattering Equations. . . . . . . . . . . 3

III Computational Program For $s_{v}\left(k^{2}, \beta\right) \ldots \ldots . . . . . . .8$

A. Approximations for $\psi_{v}$. . . . . . . . . . 8

1. The need for approximation. . . . . . . . . 8

2. Criteria for treating modes classically. . . . . 9

3. Relative magnitude of terms in $\Psi_{y} \cdot . . . . . .15$

4. Selection of significant terms in $\bar{\psi}_{v} . . . . .16$

IV Calculation of The Legendre Moments . . . . . . . . . 19

V Input Data and Other Details. . . . . . . . . . 22

VI Application to Water. . . . . . . . . . . . 28

References................. 40

Appendix: The $\mathbf{S}\left(\kappa^{2}, \beta\right)$ Function as a Fourier

Transform ............ 42

LIST OF TABLES

Table I. . . . . . . . . . . . . . . . 28

II . . . . . . . . . . . . . . . . 31

III .................... 32

IV . . . . . . . . . . . . . . . . . 35

V......................... 37

VI .................... 38

LIST OF FIGURES

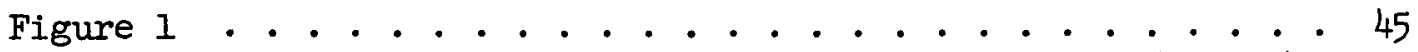

2.................... 46

3 ...................... 47

4 ..................... . . 48

5 ...................... 49 
A MACHINE PROGRAM FOR CALCULATING

SLOW NEUTRON SCATTERING CROSS SECTIONS WITH APPLICATION. TO WATER

by

H. L. McMurry and I. J. Gannon

\section{INIRODUCTION}

In refining reactor physics calculations attention has been directed toward better methods of calculating the undirected flux $\Phi(E, r)$ and the angular dependent $F \operatorname{lux} \Phi(E, \Omega, r)$ when the neutron energy $E$ is less than about $1 \mathrm{eV}$. Calculation of these fluxes requires a knowledge of the partial differential scattering cross section $\sigma\left(E_{O}, E, \underline{s}_{O}, \Omega\right)$ associated with scattering of a neutron of energy $\mathbf{s}_{\mathrm{O}}$ and direction $\Omega$ into energy $E$ and direction swen the neutron energy $E_{0}$ is below about $I \mathrm{eV}$ this cross section depends on how the scattering atoms are bound into the scattering system, and on their thermal motions $(1-\underline{3})$. It is not practical to measure $\sigma\left(E, E, \Omega_{0}, \Omega\right)$ for all values of the variables $E_{0}, E, \Omega_{0}, \Omega$ of interest, or for the variety of materials and the ranges of temperature which are met in practice. Therefore, theoretical methods for computing $\sigma$ have been sought. The best tests of such procedures are comparisons between observed and calculated values of $\sigma$, or related quantities, for pure materials under a limited range of conditions.

Two approaches have been followed. One uses a relation between $\sigma$ and the double Fourier transform of the Van Hove space-time correlation function $G(\underline{r}, t)$ (2) which, at least classically, gives the probability 
that if a particle is at $\underset{m}{r}=0$ at $t=0$ there will be one at $\underset{m}{r}$ at time t. By assuming a form for $G(\underset{m}{r}, t)$ which is a plausible representation of the way the scattering atoms move it is possible to calculate ó. The other approach, which is followed here, assumes a structure for the scattering system which is amenable to calculation. The simplest structural models which are at all reasonable depict a scattering atom as being bound in a molecular aggregate. The atom's motion is then the resultant of a vibrational motion within the aggregate together with motions of free translation and rotation of the aggregate. For gases like methane, propane, etc., the aggregate is obviously the molecule and the motion of a scattering atom can be given quite accurately. For liquids: like water it is often possible. to associate an atom with a particular molecule, but the binding between molecules must somehow be taken into account. Sometimes this can be done by assuming that the molecules exist in clusters which can: be treated.as free particles with internal degrees of vibration.

This paper describes: the basis which has been followed in developing. a program for calculating $\sigma\left(E_{O}, E, \mu\right)$ and various Legendre moments of this quantity. The equations used assume that the motion of a scattering atom can be described as arising from harmonic vibrations of the aggregate to which it. is attached, together with translational and rotational motions of the aggregate. Ways for choosing the models and describing the vibrations will be given in another paper. 


\section{THE SCATTERING EQUATIONS}

This work neglects interference scattering. Then the equation for the partial differential scattering cross section can be written in the form

$$
\sigma\left(E_{O}, E, \Omega_{0}, \Omega\right)=\Sigma_{\nu} f_{v} \sigma_{v}\left(E_{0}, E, \Omega_{0}, \Omega\right)
$$

In Eq. (I) $\sigma_{v}$ is the partial differential scattering cross section for atom $v$ associated with changes in neutron energy from $E_{0}$ into $d E$ around $E$, and of direction from $\Omega$ into d around $\Omega_{0}$. The units are usually barns/eV $x$ steradian $x$ atom, although frequently they are given per scattering molecule. If the units are per molecule $f_{v}$ is the number of atoms of type $v$ in the molecule. The type of atom means environment as well as kind of nuclide. For example, in $\mathrm{CH}_{3} \mathrm{OH}$ the hydrogens attached to the carbon are considered to be different from the one attached to the oxygen because the environments are not the same.

The equation for $\sigma_{v}$ may be written in the form (4)

$$
\left.\sigma_{v}\left(E_{0}, E, \Omega_{0}, \Omega\right)=\mid \sqrt{E / E}\right) \mid \exp \{-\beta / 2\} s_{v}\left(\kappa^{2}, \beta, \Omega_{0}, \Omega\right)
$$

In Eq. (2) $\beta=\left(E-E_{0}\right) / k T$ and $k^{2} \hbar^{2}=\left(p-p_{0}\right)^{2}$ where $p_{0}$ and $p$ are the neutron momenta before and after impact, respectively. From Eq. (2) it follows that

$$
\begin{aligned}
\sigma & =\left(\sqrt{E / E_{\Omega}}\right) \exp \{-\beta / 2\} s \\
S & =L_{\nu} v_{v} \omega_{\nu}
\end{aligned}
$$

The $S\left(\kappa^{2}, \beta, \Omega_{0}, \Omega\right)$ in Eq. (3) is the Egelstaff scattering function (4) 
which has the convenient property of being even in $\beta$. (Egelstaff extracts a factor $\sigma_{b} / 4 \pi \mathrm{kT}$ where $\sigma_{b}$ is the bound atom scattering cross section of the principal scatterer.)

In general the function $S_{v}\left(k^{2}, \beta, \Omega, \Omega\right)$ appearing in Eq. (2) will depend on how the scattering system containing atom $v$ is oriented relative to $\Omega_{0}$ and $\Omega$. Any experiment observes the average result due to scattering by systems in all orientations which are permitted.

Let $\varphi, x$, and $u=\cos \theta$ be Eulerian variables which define how a system containing atom $v$ is orjented, and let $P(u, X, \rho)$ he the probability that $\varphi$ will be in à at $\varphi, X$ in $d X$ at $X$ and 11 in All $_{\text {at. }} 1$. . Then $S_{V}$ must be computed froin*

$s_{v}\left(\kappa^{2}, \beta, \Omega, \Omega\right)=\int_{\varphi=0}^{2 \pi} \int_{x=0}^{2 \pi} \int_{u=-1}^{1} s_{v}\left(\kappa^{2}, \beta, \Omega_{0}, \Omega, x, u\right) P(\varphi, x, u) d \varphi d x d u$

When the scattering is isotropic the $s_{v}$ appearing in the integrand depends only on $\Omega_{0} \cdot \Omega=\mu$ instead of $\Omega_{0}$ and $\Omega$ separately, and this wi.l. be true also of the integrated result. In such cases it is to be expected that the scattering aggregates are oriented completely at

* The integral in Eq. (5) is like that which occurs in Eq. (23) of Reference (5). Equations (11, 12) of Reference (5) are not correct and should be replaced by a modified form of $\mathrm{Eq}$. ( $3 \mathrm{c}$ ) in which the right hand side becomes, for isotropic scattering

$$
\frac{1}{4 \pi} \int e^{-i t \alpha_{R}} e^{-\alpha_{R} t^{2}} \pi_{\tau}<0_{\nu T}^{\prime}>d u d x
$$

Here $\alpha_{R}$ is the quantity which multiplies $t$ in the exponent of Eq. (II) of Reference (5). When this, together with Eqs. (3a) and (20) are inserted in Eq. (1) of Reference (5) Eq. (23) is obtained. 
random. Then $P(\varphi, x, u)=1 / 8 \pi^{2}$ and $\int P d \varphi d X d u=1$ in Eq. (5).

The computational scheme employed here assumes the scattering is isotropic and starts with an equation for $s_{v}\left(\kappa^{2}, \beta, \mu, \varphi, x, u\right)$ in the $\operatorname{form}^{* *}(\underline{5})$

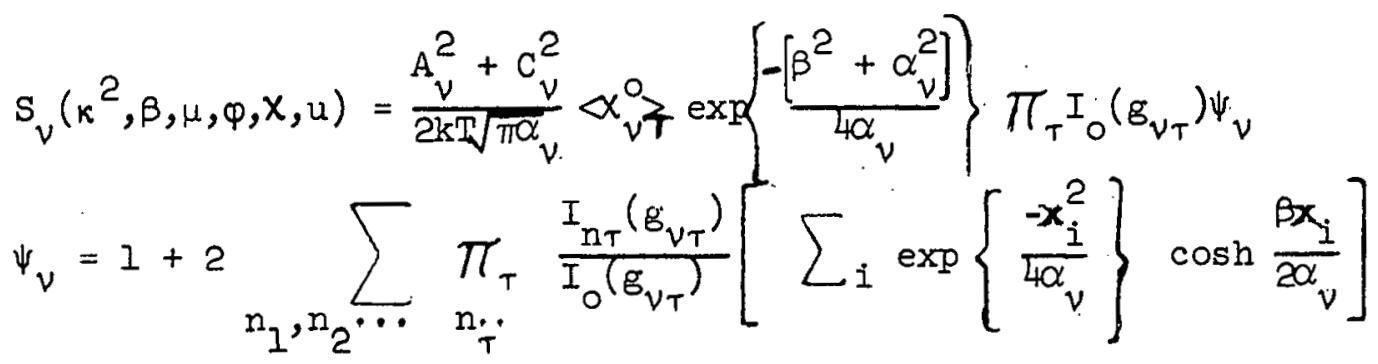

In Eq. (6) $A_{v}^{2}+C_{v}^{2}=\sigma_{b v} / 4 \pi$ where $\sigma_{b v}$ is the bound atom scattering length for atom $\nu$ and $\beta_{T}=E_{T} / k T$ where $E_{T}$ is the characteristic energy of the $\tau$ normal vibration. The function $\alpha_{\nu}$, and the arguments $g_{\nu_{\tau}}$ appearing in the modified Bessel's functions in Eq. (7), depend on quantities $\delta_{V_{T}}$ which are calculated from the transformation which gives the displacement of atom $v$ in terms of the normal coordinates $T$. The relation for a mode $T$ when $k$ is taken along the space fixed axis is (2)

$$
\delta_{V \tau}(\theta, x)=\left(A_{Z^{\prime}}^{i} T_{\nu}\right)^{2}
$$

In Eq. (8) $\underset{m v_{T}}{\mathrm{~T}}$ denotes a three element column vector with elements

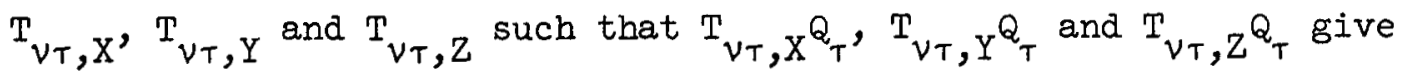
the $X, Y$, and $Z$ displacements of atom $V$, relative to axes fixed in the aggregate to which $\nu$ is attached, when the $\tau$ normal mode has the value

* The factor $\psi_{v}$ differs from Reference (5) in that the factor $\pi_{T} I_{0}\left(g_{V T}\right)$ has been taken out. 
$Q_{T} \cdot \quad$ The dependence on the Eulerian variables appears in $A_{2}^{\prime}$ which is the three element row matrix

$$
A_{\mathrm{Z}}=[-\sin \theta \cos x, \sin \theta \sin x, \cos \theta]
$$

The $\alpha_{v}$ is given in terms of the $\delta_{v \lambda}$ associated with normal modes of zero frequency, or modes which can be treated classically. Conditions under which it is justifiable to treat a normal mode classically will be given later. Such modes do not appear in $\psi_{V}$ and their influence is felt in the way they contribute to $\alpha_{v}$. The erlution for $\alpha_{v}$ is

$$
\alpha_{v}=m \beta_{0} \phi \lambda_{\gamma}^{\delta}
$$

The sum in Eq. (9) is over all modes $\lambda$ which have zero characteristic frequencies, or can be treated classically. For a gas composed of semirigid molecules the $B_{V \lambda}$ can be computed for the three translational degrees of freedom from the following $\mathbb{T}_{\mathrm{m}}$ vectors. (See Eq. 13 below.)

$$
I_{V X, T}=\frac{1}{\sqrt{M}}\left[\begin{array}{l}
1 \\
0 \\
0
\end{array}\right] ; I_{V Y, T}=\frac{1}{\sqrt{M}}\left[\begin{array}{l}
0 \\
1 \\
0
\end{array}\right] ; I_{V Z, T}=\frac{1}{\sqrt{M}}\left[\begin{array}{l}
0 \\
0 \\
1
\end{array}\right]
$$

In these relations $M$ is the mass of the entire molecule. Since ratios of $\mathrm{m} / \mathrm{M}$ ultimately appear atomic units are convenient. For the three rotational degrees of freedom the corresponding $\underset{\mathrm{T}}{\mathrm{T}}$ vectors are

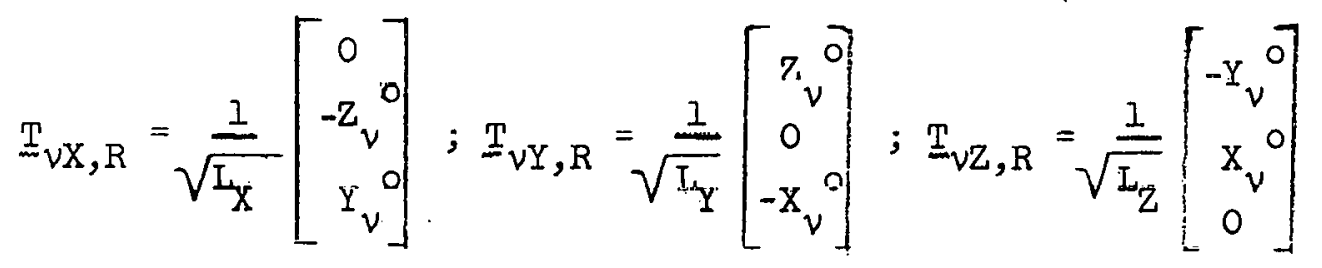

In these relations $I_{X}, I_{Y}, I_{Z}$ are moments of inertia about principal axes fixed in the molecule, and $X_{v}{ }^{0}, Y_{v}{ }^{0}$ and $Z_{v}{ }^{0}$ are the equilibrium position of atom $v$ in the molecule. When only the translational and 
rotational motions are treated classically the $\alpha_{\nu}$ computed from $\delta_{\nu \lambda}$ values obtained from these relations is the same as Eq. (26) of Reference (). In Eq. (9) $\beta_{0}=E_{0} / k T$ and $\phi=\beta / \beta_{0}+2-2 \mu\left(I+\beta / \beta_{0}\right)^{\frac{1}{2}}$. The $g_{\nu T}$, which are the arguments of the Bessel's functions appearing in Eq. (7), are defined by

$$
g_{\nu T}=\beta_{0} \phi \frac{m \delta \nu \tau}{\beta_{T} \sinh \left(\beta_{T} / 2\right)}
$$

Also, in Eq. (7) $x_{i}$ is a symbol denoting one of the sums $\Sigma_{T} \pm n_{T} \beta_{T}$ in which a particular set of signs for the $n_{T}$ are exhibited. The sum over $i$ in any term in Eq. (7) includes all combinations of signs which could lead to different values for $\Sigma_{T} \pm n_{T} \beta_{T} \cdot$ For example, if there are 5 modes involved in a term there will be 16 different combinations of signs and $16 x_{i}$ values.

Finaliy, the $\left\langle\mathbf{x}_{\nu}{ }^{0}\right\rangle_{T}$ is defined by

$$
\left\langle\boldsymbol{X}_{\nu}^{{ }^{O}}\right\rangle_{T}=\exp \left[\Sigma_{T} g_{\nu T} \cosh \left(\beta_{T} / 2\right)\right]
$$

Finally; each term in the sum in Eq. (7) involves contributions from one or more modes. The quantum numbers $n_{T}$ may have values from 0 to $\infty$. Practical devices for limiting the number of modes which contribute to any term, and for limiting the size of $\mathrm{n}_{\tau}$, will be discussed later.

At present the computational program inserts average values of the functions of $\varphi, x$, and $u$ appearing in $E q .(8)$. This leads to using (

$$
\begin{aligned}
\frac{1}{4 \pi} \iint \delta_{V T} d x d u & =\frac{T_{V T}^{\prime} T_{V T}}{3}=\frac{\bar{\delta}_{V T}}{3} \\
\bar{\delta}_{V T} & =T_{V T}^{T} T_{\nu T}^{\prime}
\end{aligned}
$$


III.. COMPUTATIONAI PROGRAM FOR: $S_{v}\left(\kappa^{2}, \beta\right)$

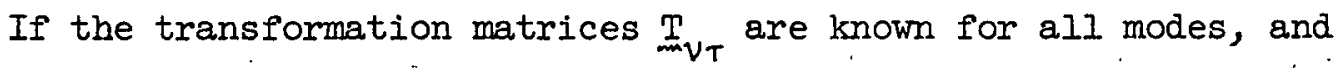
the $\sigma_{b v}$ is given, Eq. (6) provides an analytic equation for $\dot{S}_{v} \cdot$ This can be simplified by means of Eq. (12), or Eq. (5) can be integrated numerically. The results are then used in Eq. (4) to compute $\mathrm{S}$ and then Eq. (3) can be used to calculate $\sigma$. Following this the moments $\int{ }^{\prime} \sigma\left(E_{o}, E, \mu\right) P_{l}(\mu) d \mu$ may be obtained by numerical integration:

The present procedure employs Eq. $(12,13)$ and the $S_{v}, S, \sigma_{v}$, and $\sigma$ functions obtained in this way will be denoted by $\bar{s}_{v}, \bar{s}, \bar{\sigma}_{v}$, and $\bar{u}$, and the $\bar{u}_{v}$ and $y_{V \tau}$ by $\bar{\alpha}_{V}$ and $\bar{g}_{V \tau}$.

A. Approximations for $\Psi_{v}$

-1. The need for approximations

Equation (7) is an infinite series in which the number of significant terms depends: partly on the values of the characteristic vibrational energies $E_{T}$ relative to the neutron energy $E_{O} \cdot$ When $E_{0}$ is higher than one or more of the $\mathrm{E}_{T}$ the number of significant terms in $\psi_{V}$ may be very large.

For practical purposes it is necessary to use approximations which serve to limft the number of significant terms in $\psi_{V^{*}}$ : Furthermore, when this has been accomplished criteria are needed which serve to select out f'or computation only the significant terms.

In the present program the approximation which reduces the significant terms in $\Psi_{v}$ to a tractable number is one which treats modes with sufficiently low $E_{T}$ relative to $E_{0}$ in a classical manner. 
Such modes do not contribute terms to $\Psi_{v}$ their effects being felt in the way $\alpha_{v}$ is modified (see Eq. (9)).

Once this is accomplished additional criteria are used which tell what terms in $\psi_{V}$ can be significant and the actual computations are made for only these terms.

2. Criteria for treating modes classically.

If a neutron strikes an atom at rest the energy transferred to the atom is given by

$$
\frac{\left(p_{0}-p_{0}\right)^{2}}{2 m_{v}}=\frac{\kappa^{2} \hbar^{2}}{2 m_{v}}=\frac{m}{m_{v}} E_{0} \phi
$$

In Eq. (14) $m_{v}$ is the mass of the atom.

If the atom $v$ were bound in a classical molecule in which the vibrational states were not quantized Eq. (14) would still apply but the energy transferred would be distributed among the various degrees of vibrational, rotational, and translational degrees of freedom. It can be shown that for semi-rigid molecules in the gas phase $1 / m_{v}$ is given by (5)

$$
\frac{1}{m_{v}}=\Sigma_{\tau} \frac{\bar{\delta}_{v_{\tau}}}{3}
$$

In Eq. (15) the sum over $T$ includes all vibrational degrees of freedom together with rotations and translations. The $\bar{\delta}_{v \tau}$ quantities for the translational and rotational degrees of freedom are defined in connection with Eq. (9). They are calculated from Eq. (13).

When vibrations are treated classically Eq. (9) implies that the energy transferred to a molecule initially at rest is distributed 
among the various degrees of freedom in such a way that the fraction $\left(\bar{\delta}_{V \lambda} m_{v}\right) / 3$ goes to the $\lambda$ mode. This suggests that when the amount of energy which is classically transferred to the $\lambda$ mode greatly exceeds the quantum separation of levels for the mode it is justifiable to treat the mode classically. This means (after dividing by $\mathrm{kT}$ )

$$
\beta_{\lambda} \ll|\beta| \frac{\delta_{\nu \lambda}}{3} m_{v}
$$

Equation (16) is of limited utility. For one thing it does not insure that modes with $\bar{\delta}_{\nu \lambda}=0$ would be treated classically.

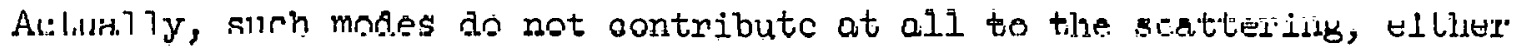
classically or quantum mechanically, and it makes no difference how they are treated. Furthermore, the mass $m_{v}$ in $E q .(16)$ is lower than is needed since modes with $E_{T} \gg E_{\lambda}$ should really not be included in Eq. (15). Finally, Eq. (16) applies in the high energy limit where the energy of the incoming neutron is so fast that the thermal motion can effectively be neglected. It takes no account of possible exchange of energy during a collision in which effects due to thermal agitation are significant. Therefore, further criteria for simplifying the calculation are desirable.

To derive conditions of this sort it is convenient to study the conditions under which the short collision time (S.C.T.) approximation (I) for calculating the cross sections is valid, and then to see under what conditions it is plausible to go clear over to the classical approximation.

When a mode $\lambda$ is treated in the short collision time approximation $\bar{\sigma}_{v}$ is calculated from ( $\underline{5}$ ) 


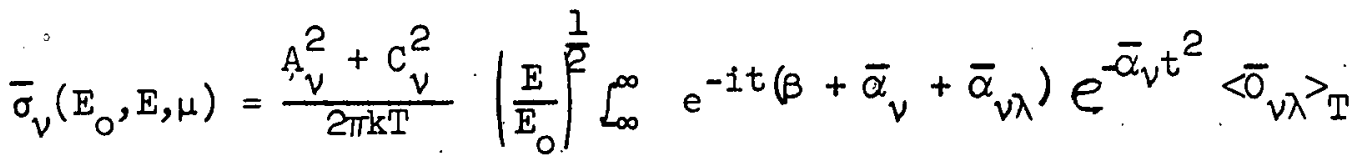

$$
\begin{aligned}
& x \pi_{T}<\bar{O}_{V_{T}^{\prime}}^{\prime}>_{T} d t
\end{aligned}
$$

In Eq. (18) the vibrational modes denoted by $T$ are assumed to be treated exactly. The corresponding operator $\left\langle\bar{O}_{V_{T}}^{\prime}\right\rangle_{T}$ is obtained from Eq. (20) of Reference (5). The operator $\left\langle\overline{0}_{V \lambda}\right\rangle_{\mathrm{T}}$ is given by (1, $\left.\underline{6}\right)$

$$
\begin{aligned}
& \left.\left\langle\overline{0}_{V \lambda}\right\rangle_{T}=\sum_{m=0}^{\infty}<\sigma_{V \lambda}^{(n)}\right\rangle_{T}(i t)^{n / n} \text { : } \\
& \left\langle\bar{o}_{V \lambda}^{(1)}\right\rangle_{T}=0 \\
& \left\langle\overline{0}_{V \lambda}^{(2)}\right\rangle_{T}=\bar{\alpha}_{V \lambda} \beta_{\lambda} \cosh \left(\beta_{\lambda} / 2\right) / \sinh \left(\beta_{\lambda} / 2\right) \text {. } \\
& \left\langle\overline{0}_{V \lambda}^{(3)}\right\rangle_{T}=-\bar{\alpha}_{\nu \lambda} \beta_{\lambda}^{2} ; \bar{\alpha}_{\nu \lambda}=\beta_{0} \Phi \bar{s}_{V \lambda} / 3
\end{aligned}
$$

When the mode $\lambda$ is treated classically Eq. (18) is the same except for replacing exp $\left(-\bar{\alpha}_{v} t^{2}\right)$. by exp $-\left(\bar{\alpha}_{v}+\bar{\alpha}_{v \lambda}\right) t^{2}$ and putting $\left\langle\overline{0}_{V \lambda}\right\rangle_{T}=1$.

The classical form of Eq. (18) will be near to the quantum calculation provided $\left\langle\overline{0}_{\nu \lambda}\right\rangle_{T} \simeq \exp \left(-\bar{\alpha}_{\nu \lambda} t^{2}\right)$ over an interval of time such that exp $-\left(\bar{\alpha}_{v}+\bar{\alpha}_{\nu \lambda}\right) t^{2}$ decays to a small value. The first three terms in the S.C.T. expansion show that this can be true only when $\beta_{\lambda} \rightarrow 0$ so that $\frac{1}{2}\left\langle\overline{0}_{v \lambda}^{(2)}\right\rangle_{\mathrm{T}} \simeq \bar{\alpha}_{v \lambda}$ and $\left\langle\overline{0}_{v \lambda}^{(3)}\right\rangle_{\mathrm{T}} \simeq 0$. Then the first three terms converge to exp $-\bar{\alpha}_{v \lambda} t^{2}$. The higher terms involve coefficients $\left\langle\overline{O_{V \lambda}}(n)_{T}\right.$ which contain powers of $\beta_{\lambda}$ and are small when $\beta_{\lambda}<1$.

This is a very stringent condition, but a useful one when low frequencies are present. Therefore, one of the criteria employed in this program is that if $\beta_{\lambda}$ is. less than or equal to a parameter $C_{4}$ 
which is between 0 and $I$ the $\lambda$ mode may be treated classically. Equation (18) will be practical for calculating the contribution of a mode $\lambda$ provided only a few terms are needed in the S.C.T. expansion. This will be the case if the time dependent terms are still small when exp $-\bar{\alpha} v t^{2}$ has declined to a small value, or if the period of the imaginary exponential factor is so short that it makes many oscillations before the S.C.T. expansion departs significantly from one. The conditions under which these requirements are satisfied may be inferred by looking at the forms of the coefficient in the S.C.T. expansion.

The coefficients in the S.C.T. expansion have been evaluated only through the $t^{3}$ terms (ㅁ) . However, the higher terms of even order, $n$, contain contributions which are proportional to $\left(\bar{\alpha}_{\nu \lambda} \beta_{\lambda}\right)^{n / 2}(\underline{6})$. These are the only terms which appear in the classical formula in which $H$ and $p_{\lambda}$ are assumed to commute. The quantum formula contains odd powers of $t$, and the expressions for the coefficients of all but the lowest powers are complicated. However, it appears reasonable to assume that when the S.C.T. approximation is valid the even powers will be of greatest importance, and in these the classical part involving $\left(\bar{\alpha}_{\nu \lambda} \beta_{\lambda}\right)^{n / 2}$ will be dominant.

If this assumption is valid conditions for insuring the adequacy of Eq. (18) may be met in several ways. First, if the time dependent terms are small when $\bar{\alpha}_{v} t^{2} \geq 1$ the requirements are met. This means

$$
\bar{\alpha}_{\nu \lambda} \beta_{\lambda} \leq c_{1} \bar{\alpha}_{v} ;\left(c_{1} \text { of the order of a few tenths }\right)(21)
$$


If $|\beta|$ is not close to $\bar{\alpha}_{\nu}+\bar{\alpha}_{v \lambda}$ the period of the exponential factor will be of the order of $\mathrm{T}=2 \pi /\left[|\beta|+\bar{\alpha}_{v}+\bar{\alpha}_{v \lambda}\right]$. If the S.C.T. expansion is still near one at this time the requirements are also met. This means

$$
\bar{\alpha}_{v \lambda} \beta_{\lambda} \leq \mathrm{c} \frac{\left[\bar{\alpha}_{\nu}+\bar{\alpha}_{\nu \lambda}+|\beta|\right]^{2}}{(2 \pi)^{2}} ; \begin{gathered}
\text { (c of the order of (22) } \\
\text { a few tenths) }
\end{gathered}
$$

The S.C.T. equation neglects anharmonic effects which are certain to be large when many quanta of vibrational energy are exchanged. Therefore, the equatior which best represents the scattering may be some compromise between Eq. (18) and the classical form where $\exp -\bar{\alpha}_{v} t^{2}$ is replaced by $\exp -\left[\bar{\alpha}_{\nu}+\bar{\alpha}_{\nu \lambda}\right] t^{2}$ and $\left\langle\overline{0}_{v \cdot \lambda}>\right.$ is put equal to one.

This program assumes that when the S.C.T. approximation is valid adequate results can be obtained by using the classical analog to Eq. (18). However, it remains to be established whether the criteria 21 , 22 are valid for stipulating that a classical treatment is tenable. Some preliminary calculations have shown that when $\mathrm{C}=0.1$ (a reasonable value) and $\bar{\alpha}_{\nu \lambda} \ll|\beta|$ (22) is not conservative enough. However, there is evidence that when $\bar{\alpha}_{\nu \lambda}>|\beta|$ (22) is over conservative. At present the program replaces (22) by the conditions

$$
\begin{aligned}
& \beta_{\lambda} \tanh \left(\beta_{\lambda} / 2\right) \leqq C_{2} \bar{\alpha}_{\nu \lambda} \\
& \beta_{\lambda} \leqq C_{3}|\beta| ; C_{3} \geqq 0.1 \quad(\text { see } p .14)
\end{aligned}
$$

with $\mathrm{C}_{2}$ and $\mathrm{C}_{3}$ the same as $\mathrm{C}$ in (22) these conditions are more conservative when $\bar{\alpha}_{v \lambda} \ll|\beta|$, but less conservative when $\bar{\alpha}_{\nu \lambda}>|\beta|$. For $\beta_{\lambda} \ll 1$ the left side of (23b) approaches $\beta_{\lambda}^{2} / 2$. This helps to eliminate the appearance of excessively large $\bar{g}_{V_{T}}$ arguments in the Bessels functions. When $\beta_{\lambda} \gg 1$ the left side approaches $\beta_{\lambda}$ and (23b) states 
that the energy $(\mathrm{kT}) \times\left(\bar{\alpha}_{V \lambda}\right)$ transferred classically to the $\lambda$ mode must be much larger than the quantum level spacing.

The use of the classical form of Eq. (18) has the practical advantage that then the equation for $\overline{\mathbf{S}}(\mathcal{k}, \beta)$ is even is $\beta$. This is not true for the S.C.T. result. Further discussion in support of using the criteria for treating modes classically is given in the Appendix.

In summary, a mode $\lambda$ is treated clasically if any of the following criteria are met

$$
\begin{aligned}
& \bar{\alpha}_{\lambda} \beta_{\lambda} \leqq c_{1}, \bar{\alpha}_{\nu} \\
& \beta_{\lambda} \tanh \left(\beta_{\lambda} / 2\right) \leqq c_{2} \bar{\alpha}_{\nu \lambda} \\
& \beta_{\lambda} \leqq c_{3}|\beta| ; c_{3} \geqq 0.1 \text { (see footnote below) } \\
& \beta_{\lambda} \leqq c_{4}
\end{aligned}
$$

Experience to date suggests that $\mathrm{C}_{1}$ must be rather small $(<0.1)$, but that $C_{2}$ and $C_{3}$ can be as high as 0.3 . However, a full evaluation of what these constants must be to insure reasonable accuracy has not been made. In most calculations $C_{4}=0$ has been used.

\footnotetext{
* When $|\beta|>\beta_{T}$ the largest term contributed by the $\tau$ mode to $\bar{\psi}_{\nu}$ is when $n_{T} \sim|\beta| / \beta_{T} \cdot$ (Compare the discussion on $p \cdot 17$ ). It is assumed that when terms with $n_{T}>10$ are required calculations can be made on a classical basis. This is the reason for the minimum value of $\mathrm{C}_{3}$ pcrmitted in (23c)
} 
3. $\therefore$ Relative magnitude of terms in $\bar{\psi}_{v} \quad \ldots \quad \therefore$.

In discussing criteria for removing terms from $\bar{\psi}_{v}$, or for

neglecting terms, it is adequate to write the general term in Eq. (7)

in the form

$$
\frac{I_{n l}\left(\bar{g}_{v 1}\right) I_{n 2}\left(\bar{g}_{v 2}\right) \cdot \cdot I_{n}\left(\bar{g}_{v N}\right)}{I_{0}\left(\bar{g}_{v 1}\right) I_{0}\left(\bar{g}_{v 2}\right) \ldots \cdot I_{0}\left(\bar{g}_{v N}\right)} \exp \frac{-x_{i}^{2}+2|\beta| x_{i}}{4 \bar{\alpha}_{v}}
$$

In Eq. (24) $x_{1}$ is one of the possible values of $\left|\Sigma_{T} n_{T} \beta_{T}\right|$ associated with quantum numbers $n_{1}, n_{2}, \cdots n_{N}$ for modës $1,2, \ldots: \because N$.

If there are $N$ modes involved in a particular term in Eq. (7) the number of $x_{i}$ associated with this term is just one-half of the sum of the number of ways having all $n_{T}$ with + signs, all but one with + signs, all but two with + signs . : ip to all with negative signs.

This gives all the possible values of $\left|\Sigma n_{T} \beta_{T}\right|$ for a given set of $n_{\tau}$ values. Thus the number of $x_{i}$ is

$\frac{N !}{2}\left[\frac{1}{N !}+\frac{1}{(N-1) !}+\frac{1}{2 !(N-2 !)} \cdot \cdot+\frac{1}{(N-2) ! 2 !}+\frac{1}{(N-1) !}+\frac{1}{\bar{N} !}\right]$ 
For example, if $N=4$ there will be 8 values and for $N=5$ there will be 16 values.

The exponential, factor in Eq. (24) is the largest when $x_{i}$ is closest to $|\beta|$. If $x_{i}=|\beta|$ the factor is $\exp \left(\beta^{2} / 4 \bar{\alpha}_{\nu}\right)$. The ratio $I_{11^{\prime} \mid}\left(\bar{g}_{V_{1}}\right) / I_{0}\left(\bar{g}_{V_{1}}\right)$ is always less than one when $n_{T}>0$ and decreases as $n_{T}$ increases. When $g_{V_{T}}<1$ the dependence is approximately

$$
\frac{I_{n \tau}\left(\bar{g}_{V \tau}\right)}{I_{0}\left(\bar{g}_{V \tau}\right)} \simeq \frac{I}{n_{\tau} !}\left(\frac{\bar{g}_{V \tau}}{2}\right)^{n_{T}}
$$

4. Selection of significant terms in $\bar{\psi}_{V}$

To be significant a term in $\bar{\psi}_{V}$ must be an acceptable fraction of the largest term. In this program the acceptable fraction is a parameter, Gl, which is part of the input.

Useful criteria for finding the largest term in $\bar{\psi}_{v}$ are hard to determine because the value of any term depends on the $x_{i}$ associated with it, and the arguments $\bar{g}_{V_{T}}$ in the Bessel's functions. The procedure used here is based on the form taken by $\bar{\psi}_{V}$ when all the $\bar{g}_{\nu \tau}$ are replaced by an average given by

$$
\bar{\delta}_{v}=\frac{3\left(M_{v}-m_{v}\right)}{M_{v} m_{v}} \cdot \frac{1}{T}
$$

In $E_{q}$. (26) $m_{v}$ is the mass of atom $v$ in atomic units and $T$ is the total number of vibrational modes which are receiving a quantum treatment. The $\mathrm{M}_{v}$ is a mass associated with the classical modes. It is calculated from Eq. (2To). Equation (26) arises from the relations ( $\underline{5})$. 


$$
\begin{aligned}
& \sum_{\tau} \frac{\bar{\delta}_{v T}}{3}=\frac{1}{m_{v}} ;(\text { sum over all modes) } \\
& \sum_{\lambda} \frac{\bar{\delta}_{v \lambda}}{3}=\frac{1}{M_{v}} ;(\text { sum over classically } \text { treated modes })(27 \mathrm{~b})
\end{aligned}
$$

It is convenient to replace $\delta_{V_{\tau}}$ in Eq. (10) by $\bar{\delta}_{V} / 3$ and to define

$$
h_{V T}=\frac{\beta_{0} \Phi}{3} \frac{m \bar{\delta}_{V}}{\beta_{T} \sinh \left(\beta_{T} / 2\right)}
$$

It is then true that if $\beta_{T}>\beta_{T^{\prime}}$

$$
I_{n}\left(h_{v \tau^{\prime}}\right)<I_{n}\left(h_{v \tau^{\prime}}\right)
$$

This makes it possible to devise relatively simple criteria for rejecting terms from $\Psi_{V}$. First the largest term is found in the series

$$
I+\sum_{\tau} \sum_{n_{T}} \frac{I_{n_{T}}\left(h_{\nu \tau}\right)}{I_{0}\left(h_{\nu T}\right)} \exp \frac{-\left(n_{T} \beta_{T}\right)^{2}+2|\beta| n_{T} \beta_{T}}{4 \bar{\alpha}_{\nu}}
$$

The largest term in this series is called the TEST term. Then the order $\mathrm{K}_{\tau}=\mathrm{n}_{\tau}$ is found for each mode such that

$$
\frac{I_{K \tau+\tau}\left(h_{\gamma}\right)}{I_{0}\left(h_{v \tau}\right)} \frac{e^{\beta^{2} / 4 \bar{\alpha}} v}{\operatorname{TEST}} \leq \mathrm{Gl}
$$

When the $\bar{g}_{\nu_{T}}$ are replaced by $h_{\nu_{T}}$ no term in $\bar{\psi}_{V}$ will exceed Gl times the TEST term if any $n_{T}>K_{T}$. It is assumed that the terms which are small in $\bar{\psi}\left(h_{V_{T}}\right)$ will also be small in $\bar{\psi}\left(\bar{g}_{V_{T}}\right)$.

A second criterion is that there exists a number $K$ such that the number of modes involved in any significant term of Eq. (7) never exceeds $\mathrm{K}$. This number is found from the relation 


$$
\frac{I_{1}\left(h_{1}\right) I_{1}\left(h_{2}\right) \cdot \cdot \cdot I_{1}\left(h_{K}+1\right)}{I_{0}\left(h_{1}\right) I_{0}\left(h_{2}\right) \cdot \cdot \cdot I_{0}\left(h_{K}+1\right)} \frac{e^{\beta^{2} / 4 \bar{\alpha}_{v}}}{\text { TEST }} \leqq G 1 .
$$

Once the $K$ and the $K_{T}$ values are found, the $\bar{\psi}_{\nu}$ is calculated using the correct $\overline{\mathrm{g}}_{V_{T}}$ values under the restrictions

(a) $n_{\tau} \leqq K_{\tau}$ in every term.

(b) Total modes involved in any term do not exceed $\mathrm{K}$. These conditions serve to eliminate many computations.

The present program is limited to values of $K$ of 5 of less. If the tests call for $K>5$, calculations are made for $K=5$ and the machine prints out KMAX exceeded and tells what atom is involved.

Finally, a condition is used which prevents calculations of any but the first term in $\bar{\psi}_{v}$ for values of $k^{2}$ where $s(k, \beta)$ is expected to be much smaller than its maximum value. The condition applies only when one or more $|\beta| / \beta_{T}>0.5$. If the contributions of a.1. but the test mode were omitted from $\bar{\psi}_{v}$, the $\bar{s}\left(\kappa^{2}, \beta\right)$ would be largest near the $\bar{\alpha}_{v}$ for which the function $\left(1 / \alpha_{\nu}\right)^{\frac{1}{2}}$

$$
\exp \cdot\left\{-\left(\beta^{2}+\bar{\alpha}_{\nu}^{2}\right) / 4 \bar{\alpha}_{\nu}-\bar{b}_{\nu} / 4\right\}
$$

is a maximum. This occurs at $\bar{\alpha}_{j,}=1+\left(1+b \beta^{2}\right)^{\frac{1}{2}}$. In these relations $b=\left[M_{\nu} \bar{\delta}_{\nu} \operatorname{coth}\left(\beta_{T} / 2\right)\right] / 3 \beta_{T}$ where $\tau$ is the test mode. The assumption is made that if the test term is less than some fraction G3 of the maximum of the above function, it is permissible to simplify by calculating only the first term in $\bar{\psi}_{v}$. (Usually this amounts to having $\bar{s}_{\nu}\left(\kappa^{2}, \beta\right)$ negligibly small.) The condition is

$$
\frac{1}{\text { TEST }} \frac{\alpha_{M}}{\bar{\alpha}_{v}} \text { el }\left[\left(1+b \beta^{2}\right)^{\frac{1}{2}} / 2-\bar{\alpha}_{v} / 4\right]_{\equiv} G 3
$$

the number $\mathrm{G} 3$ is part of the input. $\mathrm{G} 3=0.01$ appears to be satisfactory in many cases. 
For isotropic scattering where $\sigma$ depends only on $\mu=\Omega_{0} \cdot \Omega_{\mu}$, and not on the angle of defining the rotation of $\Omega$ about $\Omega_{0}$ when $\mu$ is fixed, the Lengendre moments are calculated from

$$
\bar{\sigma}_{l}\left(E_{o}, E\right)=2 \pi \int_{-i}^{1} \sigma\left(E_{0}, E, \mu\right) P_{l}(\mu) d \mu
$$

In Eq. (29) $P_{p}$ is the $l$ th Legendre polynomial and the integration over the angle $\varphi$ gives the $2 \pi$ factor.

It is convenient to express $\bar{\sigma}_{f}\left(E_{O}, E\right)$ in terms of integration over the variable $\Phi=1+E / E_{0}-2 \mu \sqrt{E / E_{0}}$. This variable is directly proportional to $\kappa^{2}$ the equation being

$$
k^{2}=\left[482.6 \mathrm{kT \beta} \beta_{0}\right] \Phi
$$

The equation for $\sigma_{f}\left(E_{0}, E\right)$ becomes

$$
\bar{\sigma}_{l}\left(E_{0}, E\right)=\pi e^{-\beta / 2} \int_{\Phi(I)}^{\bar{\Phi}(-I)} \vec{S}\left(k^{2}, \beta\right) P_{l}(\Phi) d \Phi
$$

In Eq. (3I) $P_{l}(\mu)$ is written in terms of $\Phi$ by using the relation

$$
\Phi=1+E / E_{0}-\dot{2} \mu \sqrt{E / E_{0}}
$$

The limits $\Phi(1)$ and $\Phi(-1)$ are the values of $\Phi$ in Eq. (32) when $\mu=+1$ and $\mu=-1$, respectively.

The integration of $\mathrm{Eq}$. (31) when $\beta=0$ must take account of the discontinuity in $\bar{s}_{\nu}\left(\kappa^{2}, 0\right)$ as $\bar{\Phi} \rightarrow 0$. In this limit the $\overline{\mathrm{g}}_{\nu_{\tau}} \rightarrow 0$ (see Eq. $(10)$ ) and $\overline{\mathrm{s}}_{v}\left(\kappa^{2}, 0\right) \rightarrow \operatorname{const} \hat{N}_{\bar{\Phi}}$.

It is also true that when $E_{0}$ is large $\bar{s}_{V}\left(\kappa^{2}, \beta\right)$ becomes peaked near the value of $\Phi$ which is associated with scattering by a 
classical particle at rest. For efficient numerical integration it is necessary to take the mesh size small in the region where $\bar{s}_{v}\left(k^{2}, \beta\right)$ varies rapidly with $\Phi$, and wide where the variation is slow.

This program takes care of these problems by choosing a mesh interval which is dense in the region where $\bar{\sigma}_{\nu}\left(E_{O}, E, \mu\right)$ vs $\mu$ is a maximum for the atom with largest $\bar{\sigma}_{b \nu}$. If the $\mu=\mu_{\max }$ for which $\bar{\sigma}_{\nu}$ is largest is $\leqq 0.75$ the entire range $-1 \leqq \mu \leqq 1$, is divided into $N$ equal increments. If $0.75<\mu_{\max } \leqq 0.98$ the intervals $-1 \leqq \mu<2 \mu_{\max }-1$ and $2 \mu_{\max }-1 \leqq \mu \leqq I$ are each divided into $\mathbb{N}$ parts. If $0.98 \leqq \mu_{\max } \leqq I$ the intervals $-1 \leqq \mu<0.95$ and $0.95 \leqq \mu \leqq 1$ are each divided into $\mathbb{N}$ parts. Here $N$, called MESHN in the program, determines the mesh size.

The equation for $\mu_{\max }$ was obtained by differentiating the equation for $\bar{\sigma}_{v}\left(\mathrm{E}_{0}, \mathrm{E}, \mu\right)$ assuming $\bar{\psi}_{v}=1$ in $\mathrm{Eq}$. (6). It is $\mu_{\max }=\frac{\left[\left(1-\sqrt{1+\beta^{2}} \overline{(1+4 a)} /(1+4 a)\right]+\left(2 \beta_{0}+\beta\right)(m / M)\right.}{2 \beta_{0}\left(\sqrt{1+\beta / \beta_{0}}\right)}$

In Eq. (33) $\mathrm{M}_{v}$ is the mass calculated from Eq. (27b). The quantity a is obtained by writing $\left\langle\bar{x}_{V}^{O}\right\rangle_{T}$ (see Eq. (11)) in the form $e^{-a \bar{\alpha}_{v}}$. It is given by

$$
a=\frac{\Sigma_{\tau} \bar{g}_{\nu T} \cosh \left(\beta_{T} / 2\right)}{\bar{\alpha}_{\nu}}
$$

Eq. (33) will predict $\mu_{\max }<-1$ when $\beta / \beta_{0}$ is negative and large enough in magnitude so that for the given $M_{V}$ this $\beta$ is not easily attained (and could not be reached in a collision with a particle at rest). This makes no difference, however, since the mesh spacing used for $\mu_{\max }<0.75$ is still valid.

In the computation the total thermal range from $E=0$ to some upper limit, which is now about $2 \mathrm{eV}$, is divided into several 
broad intervals each of. which is characterized by the energy group width within it: : The entire set of energy groups is numbered in sequence beginning with the one containing the energy $E=0$. The moments $\bar{\sigma}_{\ell}\left(E_{O}, E\right)$ then appear as square matrices in which the columns. are the groups for the final energy $E$, and the rows are for the. initial energy $\mathrm{E}_{0}$. Once $\bar{\sigma}_{\lambda}\left(\mathrm{E}_{\mathrm{O}}, \mathrm{E}\right)$ is computed for $\mathrm{E}<\mathrm{E}_{\mathrm{O}}$, the value $\bar{\sigma}_{\ell}\left(E, E_{0}\right)$ can be obtained by detailed balance. The program also computes $\Sigma_{i} \bar{\sigma}_{X}\left(E_{0}, E_{i}\right) \Delta E_{i}$ where $i$ is the group, $E_{i}$ the energy at the midpoint of group $i$, and $\Delta E_{i}$ is the group width. If the group structure is fine enough, this should give good results for $\bar{\sigma}_{0}\left(E_{0}\right)$. 


\section{INPUT DATA AND OTHER DETA.ILS}

The program is written in FORTRAN IV for the I.B.M. 7040 . Card types are indicated in columns 79 and 80 . Except as noted, 10 columns are allowed for each datum (making 7 data per card). Card types 0 through 6 contain input required for all options, while from type 7 on, they are specific to the various options. Integers are fixed point, others are floating point. Card Type 0

This contains the problem identification.

Card Type 1

(a) Option number. ( 1 is for $\bar{s}\left(t^{2}, \beta\right), 2$ for $\bar{\sigma}\left(E_{0}, E, \mu\right), 3$ for $\bar{\sigma}_{0}\left(E_{0}, E\right), 4$ is for $\bar{\sigma}_{l}\left(E_{0}, E\right)$ for $l=0,1,2,3$. Thus 4 also includes the information offered in 3.) Columns 1,2 (fixed point).

(b) The number of characteristic energies $E_{\tau}$. This must include modes of zero frequency like those assoclated with the translational and rotational degrees of freedom of a gas molecule. If the scattering system contains a mixture of molecules (like a gaseous mixture of $\mathrm{CH}_{4}$ and $\mathrm{C}_{3} \mathrm{H}_{8}$, or a model for water in which some molecules are in globules and others are free) the number must include all modes for all species. Columns 3-5 (fixed point).

(c) The number of scattering atoms $v$. Columns 7, 8 (fixed point).

(d). A number, called KMAX which is the largeet number of modes which can be allowed in any term is Eq. (7). The present Iimitations is KMAX $=5$. Columns 9,10 (fixed point).

(e) The absolute temperature. Columns 11-20.

(f) Gl, the number which determines whether or not terms contribute to $\bar{\psi}_{v}$. We have used GI $=0.05$, but the number is arbitrary. Columns $21-25$.

(g) G3, an index which tells the machine to circumvent calculation of $\bar{\psi}_{v}$ when $\overline{\mathrm{S}}_{v}$ is very small compared to its maximum. 
(See p. 18). Columns 25-30.

(h) $\mathrm{C}_{1}, \mathrm{C}_{2}, \mathrm{C}_{3}$ and $\mathrm{C}_{4}$, the numbers appearing in the criteria 23 a-a for treating a mode clasically. $C_{1}$ in $30-40, C_{2}$ in 40-50, $\mathrm{C}_{3}$ in 50-60 and $\mathrm{C}_{4}$ in 60-70.

(i) If a $I$ is inserted in column 77, the machine prints out the individual $\overline{\mathrm{S}}_{\nu}$ or $\bar{\sigma}_{\nu}$ contributions, a number $\mathrm{K}$ which is the largest number of modes contributing to any term $\bar{\psi}_{\nu}(\mathrm{Eq} .(7))$, together with other quantities which relate to the number of modes receiving a quantum treatment and the number appearing in $\bar{\psi}_{V}$. To understand the terminology, the method of indexing the modes must be described. First the modes, including those of zero frequency, are placed in ascending order of $\beta_{T}$. Then the modes with $\beta_{T}=0$ are put into the classical treatment and those with $\bar{\delta}_{\nu_{T}}=0$ are removed entirely from the calculation. Then the criteria (23 a-d) are applied to determine which of the remaining modes are to be treated classically. The modes which require a quantum treatment, together with those having $\beta_{T}=0$, are numbered in increasing order of $\beta_{T}$ and the following symbols are defined in terms of these numbers:

(1) NBZ denotes the first mode with non-zero $\beta_{T}$. Often only one mode has $\beta_{T}=0$ and $\mathrm{NBZ}=2$ in such cases.

(2) ITAU denotes the index of the mode with the highest $\beta_{T}$ which is calculated in $\bar{\psi}_{v}$.

(3) KTAU denotes the index of the highest mode receiving a quantum treatment. The number of modes with $\beta_{T} \neq 0$ which have been treated classically in just the total number of modes (including those with $\beta_{\tau}=U$ and $\bar{\delta}_{\nu \tau}=0$ ), involved in the problem minus KTAUU. Card Type 2

(a) This lists the characteristic energies including those which are zero. If the scattering system comprises a mixture of molecules, it is convenient to list the $\mathrm{E}_{T}$ for one molecule, then for the next, etc. However, this is not necessary, and also the $\mathrm{E}_{\mathrm{T}}$ need 
not be sequenced in order of energy. Seven, 10 column entries per card. Card Type 3

(a) This gives the fractions of atoms of type $\nu$. These are the $f_{v}$ factors. If the scattering is for a molecule, $f_{v}$ will be the number of atoms of type $v$ in the molecule. Seven per card.

Card Type 4

(a) This gives the value of $\sigma_{b \nu} / 4 \pi$ for each atom. The order of listing must be the same as the order of atoms for the $f_{v}$ on card 3.

Card Type 5

(a) This gives the mass, in atomic units, of atoms $v$ listed in the order of atoms on card 3. The program assumes a neutron mass of 1.0 . If the exact $\mathrm{m} / \mathrm{M}_{v}$ ratio is desired this must be remembered. Card Type 6

(a) The type 6 cards give the $\bar{\delta}_{V \tau}$ values for each atom and each mode. The following rules must be observed:

(I) The $\bar{\delta}_{V_{\tau}}$ are listed for the atoms in the order of their appearance on card 3.

(2) Each atom has its own type 6 cards. That is, data for one atom must not be included on a card for another atom.

$$
\text { (3) For each atom the } \bar{\delta}_{V_{\tau}} \text { must be in the order of } E_{\tau}
$$
listed on Card Iype 2. There must be a $\bar{\delta}_{V_{T}}$ for every mode $T$, although they are sometimes zero. As an example, if the scattering is by a mixture of $\mathrm{CH}_{4}$ and $\mathrm{C}_{3} \mathrm{H}_{8}$ molecules it would be most convenient to list on card 2 first the $E_{T}$ for one molecule ( say $\mathrm{CH}_{4}$ ), then for the other. Then on card 6 the $\bar{\delta}_{V_{\tau}}$ for one of the hydrogens on $\mathrm{CH}_{4}$ would have zero values for all of the $27 \mathrm{C}_{3} \mathrm{H}_{8}$ modes. However 
the zeros would have to be listed for these 27 modes. It must be remembered that modes with $\mathrm{E}_{\tau}=0$, such as those associated with free translations and rotations, mast have $\bar{\delta}_{V_{T}}$ entries. (See after Eq. (9)). The remaining card types pertain to specific options. They are: Option $i, \bar{s}\left(\kappa^{2}, \beta\right)$ Card Type 7

(a) The minimum $\kappa^{2}$. Columns $1-10$.

(b) The maximum $k^{2}$. Columns $11-20$.

(c) A number 62 which determines successive $k^{2}$ values to be calculated through the relation $k_{i+1}^{2}=(1+G 2) k_{i}^{2}$. Columns $21-30$.

(d) An arbitrary mass. Often $\overline{\mathrm{S}}\left(\kappa^{2}, \beta\right)$ is plotted against an $\alpha$ instead of $\kappa^{2}$. A mass must be used to define $\alpha$ and this is what appears in this entry. Columns $31-40$.

(e) The number of $\beta$ values for which $\overline{\mathbf{s}}\left(\kappa^{2}, \beta\right)$ vs $\kappa^{2}$ calculations are to be made. Columns 4l-50 (fixed point). Card Type 8

(a) This lists the $\beta$ values to be calculated. Seven per card. Option 2, $\bar{\sigma}\left(E_{0}, E, \mu\right)$ Card Type 9.

(a) The value of $E_{0^{\circ}}$ Columns 1-10.

(b) The minimum $E$ value. Columns 11-20.

(c) The increment $\Delta \mathrm{E}$ between $\mathrm{E}$ values. Columns 2l-30.

(d) The maximum E value. Columns $31-40$.

(e) The arbitrary mass (same as under Card Type 7 in item a). Columns 41-50.. .

(f) The number of $\mu$ values for which $\bar{\sigma}(\mathbb{E}, E, \mu)$ vs $E$ 
calculations are desired. (Columns 51-60 (rixed point). Card Type 10

(a) The $\mu$ values are given. Seven per card.

Option 3, $\bar{\sigma}(\mathrm{E}, \mathrm{E})$

Card Type 11

(a) The number of $E_{0}$ values to be calculated. 1-10 (fxt. pt.)

(b) The number of $\mathrm{E}$ values. Columns $1.1-20$ (fixed point).

(c) A number of MESHN which is the number of mesh intervals the integration ranges in $\Phi$ are to be divided into. 21-30 (fixed point)

(d) The index of the principal scatterer. This is generally the atom with the highest $\sigma_{b v} / 4 \pi$ on Card Type 4 . Cols. 3l-40 (fxt. pt.)

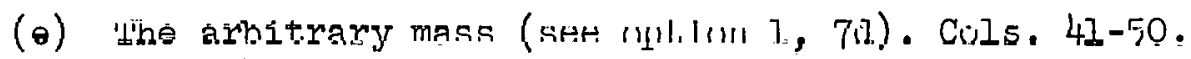

Card Type 12

(a) The $E_{0}$ values are given. Seven per card.

Card Type 13

(a) The $E$ values are given. Seven per card.

Option 4, The Legendre Moments

Card Type 14

(a) A number NCD which gives the number of type 15 cards. The type 15 cards relate to how the range from $E=0$ to some upper limit (now around $2 \mathrm{eV}$ ) is to be divided. This range is first divided into several rather broad intervals which are given NCD indexes in the order of increasing energy. The intervals are characterized by the different sizes of group spacings within the intervals. An important item is the numbering of theee small energy subdivisions. Their indexes $N E i$ are in increasing order of energy. This is used in the definitions below. The maximum NE'i allowed is 32 . Columns 1-10 (fixed point). 
(b) A number NEI which tells the energy group for the lowest energy $E$ to be used in the integration. Thus, when $\bar{\sigma}_{f}\left(E_{\circ}, E\right)$ is computed, there may be reasons why values of $\mathrm{E}$ lower than some value specified by NEl need not be computed. Columns 1l-20 (fixed point).

(c) A number NE 2 which gives the group index for the highest energy $E$ which is to be calculated. Columns $21-30$ (fixed point)

(d) A number denoted by $\mathrm{NEOI}$, which is the group index for the lowest $\mathrm{E}_{\mathrm{O}}$. Calculations are made for this and all higher energy values. Columns $31-40$ (fixed point).

(e) MESHN. Same as on Card Type 11, item c. It determines the interval size for the $\Delta \Phi$ integration. Cols. 4l-50 (fixed point). (f) The index of the principal scatterer (see Card Type 1l, item d). Columns 5I-60 (fixed point).

(g) An identification number which can be used to designate what problem is being solved. Columns 61-70 (fixed point).

(h) The arbitrary mass (option 1, 7d). Columns 71-77.

Card Type 15

The number of type 15 cards is specified by the NCD in 14 (a). There is a type 15 card for each broad energy interval containing the following information.

(a) The number of subgroups, NEOK, into which this broad energy interval is divided. Columns $1-10$ (fixed point).

(b) The midpoint, EOK, of the lowest energy group in this broad interval. Columns $11-20$.

(c). The group width, $\Delta E$; in thi.s broad intervals. Columns $21-30$.

* The total number of energy groups cannot exceed 32 . 


\section{APPLICATION TO WATER}

To illustrate the program, computations are given for two models which attempt to explain the scattering by water. The simplest model is one due to Nelkin (7) which has been used with some success for calculating quantities, such as spectra, which depend on integrals over energy and scattering angle of functions involving the partial differential scattering cross sections. These quantities are not likely to be very sensitive to the choice of model. Table I gives the input parameters used in the Nelkin model. A temperature of' $293^{\circ} \mathrm{K}$ is assumed. The ${ }^{8} \delta_{V}$ values associated with the zero trequency are the sum of those for the translational degrees of freedom of the free molecule. (See after Eq. (a)) and give $\mathrm{M}_{\mathrm{H}}=\mathrm{M}_{\mathrm{O}}=18$.

\section{TABLE I}

Parameters for Nelkin Model

$\begin{array}{lllll}E_{T} & 0 & 0.06 & 0.2 & 0.46 \\ \beta_{T} & 0 & 2.4 & 8.0 & 18.4 \\ \bar{\delta}_{H_{T}} & 0.1667 & 1.4233 & 0.45 & 0.96 \\ \bar{\delta}_{O_{T}} & 0.1667 & 0.0093 & .002 l_{1} & .0091\end{array}$

$\bar{S}\left(\kappa^{2}, \beta\right)$ vs $\beta$ curves calculated on the Nelkin model are compared with data of Haywood ${ }^{(8)}$, in Figure 1 . The agreement is mediocre. The curves with $\beta>0.2$ fall off too rapidly as $k^{2}$ decreases, except that for $\beta=2.4$ which is in resonance with the $0.06 \mathrm{eV}$ torsional mode. The maxima of these curves are higher than the experimental data. These defects arise be cause the Nelkin model does not provide any modes with very low $\beta_{T}$, and the effective mass for the hydrogen is too low. 
The Nelkin model. treats the translations as free and allows for but one torsional frequency. However, it is.highly likely that hydrogen bonding between water molecules results in the appearance of dimers, trimers and higher polymers $(9)$. Therefore, it is reasonable to assume that water is composed of aggregates of $\mathrm{H}_{2} \mathrm{O}$ molecules and these exhibit a large number of vibrational frequencies.

The experimental $\bar{S}\left(\kappa^{2}, \beta\right)$ ve $k^{2}$ curves provide a clue as to what sort of model is needed. When these curves are plotted for increments in $\beta$ of 0.1 up to $\beta=1.0$, none of them cross and they decrease rather slowly as $\kappa^{2}$ decreases below $\kappa^{2}=10$. This implies that the model for the calculations should have a rather large number of modes in the range of $0<\mathrm{E}_{\mathrm{T}}<.025 \mathrm{eV}$. An idea of the number required can be obtained by noting that if $\bar{S}\left(k^{2}, \beta\right)$ is plotted against $\beta$ for a fixed $\kappa^{2}$, the contribution of the $\tau$ mode varies roughly as $\exp \cdot\left[-\left(\beta-\beta_{T}\right)^{2} / 4 \bar{\alpha}_{H}\right]$. This function has a half width given by

$$
\therefore \frac{\beta_{\frac{1}{2}}}{=} 4 \bar{\alpha}_{\mathrm{H}}^{\frac{1}{2}} \operatorname{Ln} 2=0.8\left[\frac{k^{2}}{M_{\mathrm{H}}}\right]^{\frac{1}{2}} ;\left(\text { at } \mathrm{T}=293^{\circ} \mathrm{K}\right)
$$

If the $\bar{S}\left(\kappa^{2}, \beta\right)$ vs $\kappa^{2}$ curves are not to cross the spacing between $\beta_{T}$ values in the range $0<\beta_{T}<1.0$ should be no larger than this half width. For $k^{2}=2.0$ and $\mathrm{M}_{\mathrm{H}}=100$, this gives a value of about 0.1 for the spacing between $\beta_{T}$ values.

A model with a large number of vibrational modes can be constructed on the assumption that all water molecules are in the same environment. A more general treatment would allow for ditt'erences in the sizes of the aggregates, and in the locations of the water molecules within an aggregate. The first approach assumes that ail 
$H$ atoms scatter alike and partake of the same frequencies, $E_{T}$, and amplitude factors $\bar{\delta}_{\mathrm{H}_{T}}$. The second assumes a mixture of aggregates each with its own set of characteristic frequencies and amplitude factors.

A number of models were tried. The computation time increases rapidly with the number of modes assigned to an aggregate. The best agreement so far obtained assumes a single aggregate with 17 non-zero frequencies and an effective hydrogen mass of 150 . However, the computation time was large and to reduce this to a practical range these modes were assumed to be distributed among three types of aggregates, none of which had more than 8 frequencies. Tables II and III are reproductions of input data for the IBM 7040 for the two cases considered. The characteristic energies given on the Type 2 cards in Table III are in increasing order of $E_{T}$ for each aggregate, and occur in three groups, one for each aggregate. The $f_{v}^{\prime}$ values Listed on card type 3 are chosen so that the cross sections are in barns per $\mathrm{H}_{2} \mathrm{O}$ molecule. Then $f_{H}=2 f_{O}$ for each aggregate. The first two entries give $f_{H}=0.5$ and $f_{O}=0.25$ for aggregate 1 . The 0.25 value means that $25 \%$ of the aggregates are of this type. Thus the fractions associated with each aggregate are given by the $f_{0}$ factors

Finally, there is a set of Type 6 cards for each atom in each aggregate, the order being the same as the order of atoms on card 3. Thus the first four Type 6 cards contain the $\delta_{H_{T}}$ values for the first aggregate, the third set of four Type 6 cards contain $\overline{\mathrm{S}}_{\mathrm{H}_{T}}$ values for the second aggregate, etc. For any aggregate, they occur in the same sequence as the $E_{T}$ on the 2 cards.

It is seen that there is large flexibility in the choice of $E_{T}$ 


\section{TABLE II}

Input Data For Calculating $\overline{\mathrm{S}}\left(\boldsymbol{k}^{2}, \beta\right)$ For Water

Assuming Large Aggregates With 17 Modes

(Title Card Not Shown)

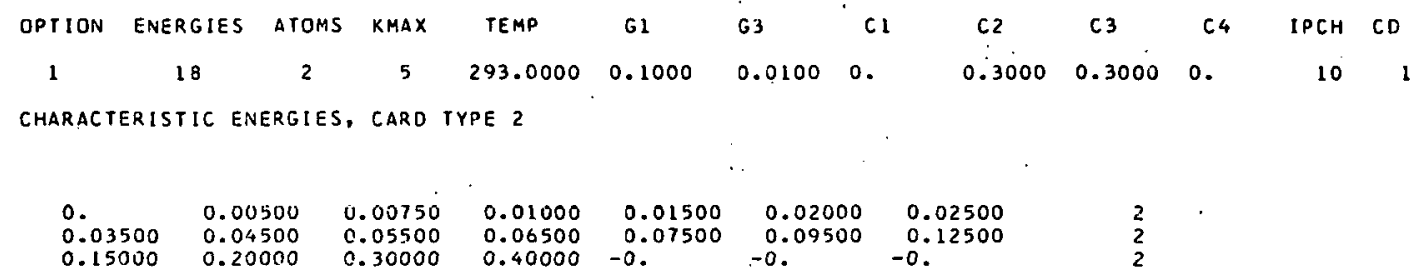

FRACTIONS OF ATOM NU, CARD IYPE 3

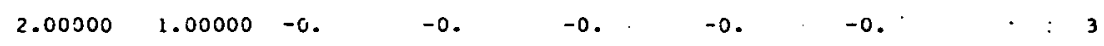

SCATTERING AMPLITUOES FOR ATOM NU $(A 2+C 2)$, CARD TYPE 4

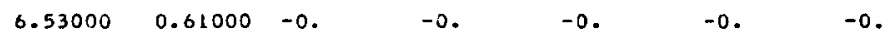

MASS OF ATOM, CARD TYPE 5

-0 .

$-0$.

$-0$

$-0$

5

AMPLITUDE FACTORS DELTAINU, TAU), CARO TYPE 6

\begin{tabular}{|c|c|c|c|c|c|c|c|}
\hline $\begin{array}{l}0.02000 \\
0.07000 \\
0.30000 \\
0.02000 \\
0.00600 \\
0.00100\end{array}$ & $\begin{array}{l}0.02400 \\
0.15000 \\
0.40000 \\
0.02000 \\
0.00700 \\
0.00150\end{array}$ & $\begin{array}{l}0.02400 \\
0.20000 \\
0.40000 \\
0.03000 \\
0.00800 \\
0.00400\end{array}$ & $\begin{array}{l}0.02200 \\
0.25000 \\
0.43000 \\
0.03000 \\
0.00800 \\
0.00500\end{array}$ & $\begin{array}{l}0.07000 \\
0.20000 \\
-0 . \\
0.01000 \\
0.00700 \\
-0 .\end{array}$ & $\begin{array}{l}0.03000 \\
0.15000 \\
-0 . \\
0.00800 \\
0.00600 \\
-0 .\end{array}$ & $\begin{array}{l}0.06000 \\
0.20000 \\
-0 . \\
0.00500 \\
0.01100 \\
-0 .\end{array}$ & $\begin{array}{l}6 \\
6 \\
6 \\
6 \\
6 \\
6\end{array}$ \\
\hline KAP MIN & KAP MAX & G2 & . & ARB. MASS & & NBETA & CARD 7 \\
\hline 1. 000ல0ல & 100.00000 & 0.7000 & & 00000 & 11 & & 7 \\
\hline
\end{tabular}

Beta VALUES For KaPPa SQUareo CALCULATION, CARO 8

0.60000
-0.

0.80000
-0.

1.00000
-0.

8
8 
Input Data For Calculating $\bar{s}\left(k^{2}, \beta\right)$. For Water

Assuming A: Mixture of Three Types of Aggregates

(Title Card Not Shown)

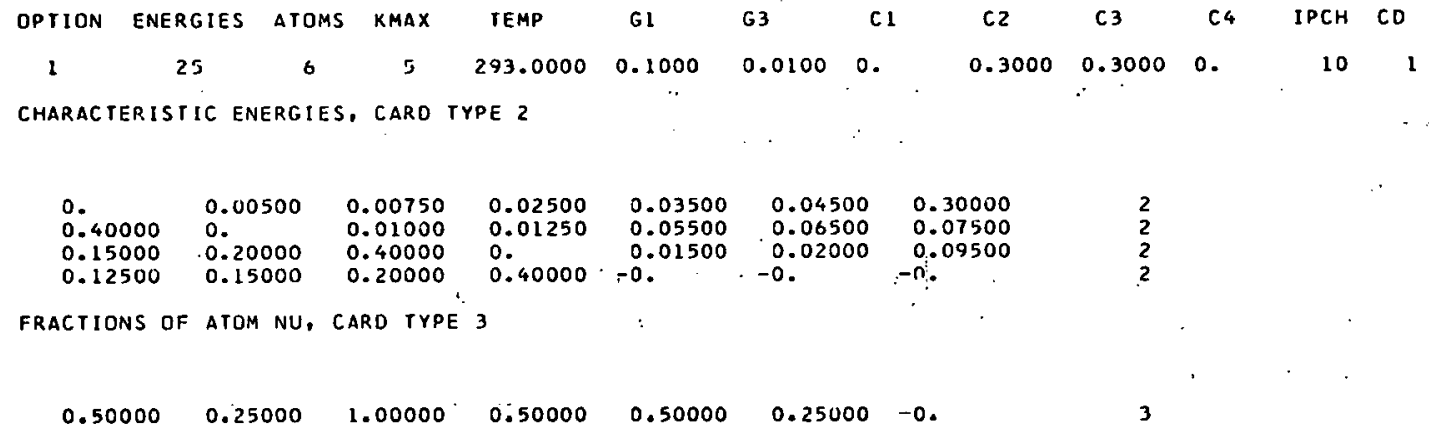

SCATTERING AMPLITUDES FRR ATOM NU $(A 2+C 2)$, CARD TYPE 4

$\begin{array}{lllllll}6.53000 & 0.61000 & 6.53000 & 0.61000 & 6.53000 & 0.61000 & -0 .\end{array}$

MASS RF ATIM, CARD TYPF, 5

$\begin{array}{llllllll}1.00000 & 16.00000 & 1.00000 & 16.00000 & 1.00000 & 16.00000 & -0 & 5\end{array}$

AMPLITUDE FACTORS DELTAINU, TAU), CARD TYPE 6

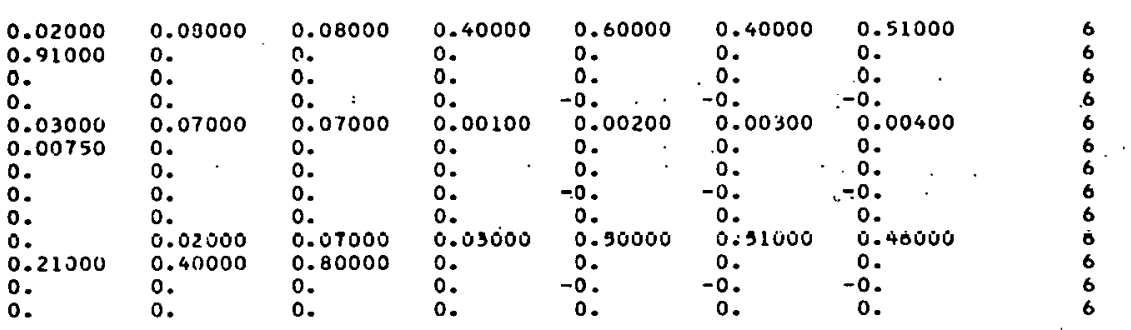

\begin{tabular}{|c|c|c|c|c|c|c|c|}
\hline $\begin{array}{l}0 . \\
0: 010100 \\
0 . \\
0 . \\
0 . \\
0 . \\
0.82000 \\
0 . \\
0 . \\
0.0 \\
0.00300\end{array}$ & $\begin{array}{l}0.03000 \\
0.010300 \\
0 . \\
0 . \\
0 . \\
0 . \\
0.31000 \\
0 . \\
0 . \\
0 . \\
0.00200\end{array}$ & $\begin{array}{l}0.07000 \\
0.0 .750 \\
0 . \\
0 . \\
0 . \\
0 . \\
0.30000 \\
0 . \\
0 . \\
0 . \\
0.00200\end{array}$ & $\begin{array}{l}0.07000 \\
0 . \\
0 . \\
0 . \\
0 . \\
0.02000 \\
0.80000 \\
0 . \\
0 . \\
0.02000 \\
0.00750\end{array}$ & $\begin{array}{l}0.00100 \\
0 . \\
-0 . \\
0 . \\
0 . \\
0.10000 \\
-0 . \\
0 . \\
0 . \\
0.10000 \\
-0 .\end{array}$ & $\begin{array}{l}0.00200 \\
0 . \\
-0 . \\
0 . \\
0 . \\
0.05000 \\
-0 . \\
0 . \\
0 . \\
0.05000 \\
-0 .\end{array}$ & $\begin{array}{l}0.00300 \\
0 . \\
-0 . \\
0 . \\
0 . \\
0.00000 \\
-0 . \\
0 . \\
0.0 \\
0.00300 \\
-0 .\end{array}$ & $\begin{array}{l}6 \\
6 \\
6 \\
6 \\
6 \\
6 . \\
6 \\
6\end{array}$ \\
\hline KAP MIN & KAP MAX & G2 & & ARB. MASS & & NBETA & CARD 7 \\
\hline 1.00000 & 100.00000 & 0.7000 & .1. & 00000 & 13 & & 7 \\
\hline
\end{tabular}

BEta VALUES F́OR KAPPA SQUARED CALCULATION, CARD 8

$\begin{array}{llllllll}0 . & 0.20000 & 0.30000 & 0.40000 & 0.50000 & 0.60000 & 0.80000 & 8 \\ 1.00000 & 1.40000 & 2.00000 & 2.40000 & 3.00000 & 5.00000 & -0 . & 8\end{array}$


values. There is similar flexibility in assigning $\bar{\delta}_{H_{T}}$ and $\bar{\delta}_{O_{T}}$ values. However, several rules restrict the choices of the $\dot{\delta}_{\nu_{T}}$ considerably. First, it must always be true that

$$
\begin{aligned}
& \Sigma_{T} \bar{\delta}_{\mathrm{II}_{T}}=3.0 \\
& \Sigma_{T} \bar{\delta}_{\mathrm{O}, T}=0.1875
\end{aligned}
$$

Sum over all modes

These are the relations in Eq. (27a). A second condition used here is that for the modes, in the range $0 \leqq \mathrm{E}_{\mathrm{T}}<0.025 \mathrm{eV}$.

$$
\begin{aligned}
& \Sigma_{T} \bar{\delta}_{H_{T}}=0.167 \\
& \Sigma \bar{\delta}_{O, T}=0.167
\end{aligned}
$$

Sum over low frequency modes

The only justification for this is that if these modes are associated with translational oscillations it is reasonable to assume that their $\bar{\delta}_{V \tau}$ values have a sum comparable to those for the translational degrees of freedom of the free molecule. The third condition is that for the higher frequency modes with $0.025<\mathrm{E}_{T}<0.15 \mathrm{eV}$

$$
\begin{aligned}
& \Sigma_{T} \bar{\delta}_{H_{T}}=1.42 \\
& \Sigma_{T} \bar{\delta}_{O T}=0.006
\end{aligned}
$$

$$
\text { Modes in } .025<\mathrm{E}_{\mathrm{T}}<0.15 \mathrm{eV} \text { range. }
$$

These values are those appropriate to the rotational degrees of freedom of the free molecules. This leaves for the modes with $0.15<\mathrm{E}_{\mathrm{T}}$ the condition

$$
\begin{aligned}
& \Sigma \overline{\mathrm{S}}_{\mathrm{H}_{T}}=1.413 \\
& \Sigma \overline{\mathrm{S}}_{\mathrm{O}, \tau}=0.0145 \therefore
\end{aligned}
$$$$
\text { Modes with } 0.15<\mathrm{E}_{\boldsymbol{T}}
$$

In the free molecule, these values are associated with the three internal vibrational modes of $0.2,0.45$, and $0.46 \mathrm{eV}$.

These guide lines have been used assigning $\bar{\delta}_{H_{T}}$ and $\bar{\delta}_{O_{T}}$ values 
to the modes in the models which have been tried.

Experimental $\bar{S}\left(k^{2}, \beta\right)$ vs $\kappa^{2}$ curves are compared with calculation based on these models in Figs. 2 and 3. Not much effort has been expended on getting an optimum set of $\mathrm{E}_{\tau}$ and $\bar{\delta}_{\nu^{\top}}$ values. However, the agreement shown in Figs. 2 and 3 is better than on the Nelkin model, and improvement should be possible.

Figure 4 shows calculations of $\bar{\sigma}\left(E_{0}, E, \mu\right)$ vs $E$ for $E_{0}=0.025 \mathrm{eV}$ and $O=90^{\circ}$ using the Nelkin model, and on the three molecule model. It is seen that the lower $M_{\text {II }}$ used in the Nelkin model results in a much broader curve.

Figure 5 shows $\bar{\sigma}\left(E_{0}, E\right)$ vs E curves calculated using the Nelkin and 3 aggregate models for a neutron energy $\mathrm{E}_{\mathrm{O}}=0.025$. A mesh interval of 10 was used in the integration. Table IV compares total cross sections $\bar{\sigma}\left(E_{0}\right)$ calculated using these two models for several vâlues of $\mathrm{E}_{\mathrm{O}}$ wìth the expérimental data from BiṽL $33^{(1.0)}$. 'line calculated values were obtained by integrating the $\bar{\sigma}\left(\mathrm{E}_{\mathrm{O}}, \mathrm{E}\right)$ vs $\mathrm{E}$ curves with a planimeter. The agreement is very good for both models.

To obtain an estimate of the time to compute a matrix of moments $\bar{\sigma}_{\rho}\left(E_{0}, E\right)(E q .(29))$ a calculation was made in which the range $0-0.0125 \mathrm{eV}$ was divided into 5 increments. The $q\left(E_{0}, E\right)$ for the $5 \times 5$ matrix was computed in about 1 minute on the Nelkin model, and in 4.2 minutes on the 3 aggregate model. This indicates that about $2-1 / 2$ hours might be required to compute a $32 \times 32$ matrix using the 3 aggregate model. However, this estimate takes no account of the fact that elements which are far off the diagonal may be computed using simpler approximations than were required in this test problem. Thus the 2-1/2 hour estimate may be high. 


\section{Comparison of Calculated With Observed Total Scattering Cross Sections}

Total Scattering Cross Section (barns/molecule)

$\frac{E_{0}(e V)}{0.005}$
0.025
0.05

Observed

Melkin

165

170

3 Aggregate

107

104

Mixture

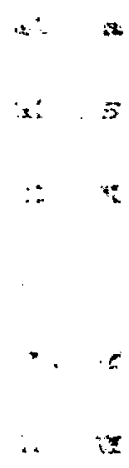




\section{OUTPUT FORMAT}

Typical output data for calculations of $\bar{S}\left(\kappa^{2}, \beta\right)$ vs $\kappa^{2}$, and $\bar{\sigma}_{l}\left(E_{0}, E\right)$ are given in Tables $V$ and $V I$. In Table $V$ a, $I$ in column 77 of card 1 instructed the machine to print out the contribution from each atom. The $\bar{S}\left(k^{2}, \beta\right)$ are given for $\beta=3.0$ on the three aggregate model. The last 7 columns contain useful information which bears some explanation. The column headed MASS gives the effective mass $\mathrm{M}_{\nu}$. Because $\mathrm{C}_{3}=0.3$ modes, with $\beta_{\tau}<0.15$ are treated classically. For this reason the values of $\mathrm{M}_{\mathrm{H}}$, which are those for atoms $1,3,5$ as indicated in the columr headed $\mathbb{N U}$, are less than the value of 150 which prevails for the low $\beta$ curves. Whenever, ITAU $=0$, it means that with $\mathrm{G} 3=0.01$ the calculation of terms in $\bar{\psi}_{v}$ has been circumvented. This is true for the oxygen atoms (atoms 2, 4,6) for all $\kappa^{2}$, and for the lower $\kappa^{2}$ values it is true for the $\mathrm{H}$ atoms. Circumventing the calculation of terms in $\bar{\psi}_{\nu}$ saves much computing time without significant cost in accuracy. The number NBZ is the index for the lowest mode receiving a quantum treatment, and ITAU - NBZ +1 tells how many modes contribute terms to $\bar{\psi}_{v}$. The number under $K$ tells the highest order of the Bessels function used in $\bar{\psi}_{V}$. When ITAU - NBZ $+I$ and $K$ are large, many modes are involved and the computing time is large. These terms are described in more detail in Part $\mathrm{V}$ under Card Type 1.

Part of the print out for option $4, \bar{\sigma}_{l}\left(E_{o}, E\right)$ for the $5 \times 5$ matrix calculation referred to above, is given for the three aggregate model in Table VI because it merits some explanation. The line which starts with the word TOTALS gives first the value of $E_{0}$, then in successive 
TABLE V

Typical Output of $\bar{s}\left(k^{2}, \beta\right)$ vs $k^{2}$ For 3 Aggregate Model (Detailed Printout is Shown)

\begin{tabular}{|c|c|c|c|c|c|c|c|c|c|c|c|}
\hline DR $S(N U)$ & KAPPA $S$ & BOPHI & BETA & $A L F A-R A L F A$ & MASS & NB2 & ITAU & k & NU & KTAU & NTMX \\
\hline 0. & 1.00000 & 0.08207 & 3.000 & 3.00492 & 16.667 & 4 & 0 & 0 & 1 & 8 & 2 \\
\hline 0. & 1.00000 & 0.08207 & 3.000 & 3.00465 & 17.647 & 4 & 0 & 0 & 2 & 8 & 2 \\
\hline 1.17786 & 1.00000 & 0.08207 & 3.000 & 0.00328 & 25.000 & 4 & 7 & 1 & 3 & 9 & l \\
\hline 0 & 1.00000 & 0.08207 & 3.000 & 3.00465 & 17.647 & 4 & 0 & 0 & 4 & 9 & 1 \\
\hline 0. & 1.00000 & 0.08207 & 3.000 & 0.00465 & 17.647 & 4 & 0 & 0 & 5 & 8 & 1 \\
\hline 0. & 1.00000 & 0.08207 & 3.000 & 0.00465 & 17.647 & 4 & 0 & 0 & 6 & a & 1 \\
\hline $\begin{array}{l}1.17786 \\
0.05059\end{array}$ & $\begin{array}{l}1.000 n n \\
1.70000\end{array}$ & $\begin{array}{l}0.08207 \\
0.13953\end{array}$ & 3.000 & $\begin{array}{l}0.08207 \\
0.00837\end{array}$ & 16.667 & & & & & & 2 \\
\hline 0.05059 & $\begin{array}{l}1.70000 \\
1.70000\end{array}$ & 0.13953 & 3.000 & 0.00791 & $\begin{array}{l}10.001 \\
17.647\end{array}$ & $\begin{array}{l}4 \\
4\end{array}$ & $\begin{array}{l}7 \\
0\end{array}$ & $\begin{array}{l}3 \\
0\end{array}$ & 1 & 8 & 2 \\
\hline $\begin{array}{l}0 . \\
1.55287\end{array}$ & 1.70000 & 0.13953 & 3.000 & 3.00558 & 25.000 & 4 & 7 & 1 & 3 & $\stackrel{n}{q}$ & $i$ \\
\hline 0. & 1.70000 & 0.13953 & 3.000 & 0.00791 & 17.647 & 4 & 0 & 0 & 4 & 9 & I \\
\hline 0. & 1.70000 & 0.13953 & 3.000 & 0.00791 & 17.647 & 4 & 0 & 0 & 5 & 8 & 1 \\
\hline 0. & 1.70000 & 0.13953 & 3.000 & 0.00791 & 17.647 & 4 & 0 & 0 & 6 & 8 & 1 \\
\hline 1.57816 & 1.70000 & 0.13953 & 3.000 & 0.13953 & & & & & & & \\
\hline 0.17797 & 2. 89000 & 0.23719 & 3.000 & 0.01423 & 16.667 & 4 & 7 & 3 & 1 & 8 & 2 \\
\hline 0. & 2.89000 & 0.23719 & 3.000 & 0.01344 & 17.647 & 4 & 0 & 0 & 2 & B & 2 \\
\hline 2.02740 & 2.89000 & 0.23719 & 3.000 & 0.00949 & 25.000 & .4 & 7 & i & 3 & 9 & $i$ \\
\hline 0 . & 2.89000 & 0.23719 & 3.000 & 3.01344 & 17.647 & 4 & 0 & 0 & 4 & 9 & i \\
\hline o. & 2.89000 & 0.23719 & 3.000 & 0.01344 & 17.647 & 4 & 0 & 0 & 5 & B & l \\
\hline 0. & 2.89000 & 0.23719 & 3.000 & 0.01344 & 17.647 & 4 & 0 & 0 & 6 & B & 1 \\
\hline 2.11639 & 2.89000 & 0.23719 & 3.000 & 0.23719 & & & & & & & \\
\hline 0.50265 & 4.91300 & 0.40323 & 3.000 & 0.02419 & 16.667 & 4 & 6 & 3 & 1 & 8 & 2 \\
\hline 0. & 4.91300 & 0.40323 & 3.000 & 0.02285 & 17.647 & 4 & 0 & 0 & 2 & $\boldsymbol{\theta}$ & 2 \\
\hline 2.75665 & 4.91300 & 0.40323 & 3.000 & 0.01613 & 25.000 & 4 & 7 & 1 . & 3 & 9 & 1 \\
\hline 0 . & 4.91300 & 0.40323 & 3.000 & 0.02285 & 17.047 & 4 & 0 & 0 & 4 & 9 & i \\
\hline 0. & 4.91300 & 0.40323 & 3.000 & 0.02285 & 17.647 & 4 & 0 & 0 & 5 & B & 1 \\
\hline 0. & 4.91300 & 0.40323 & 3.000 & 0.02285 & 17.647 & 4 & 0 & 0 & 6 & B & 1 \\
\hline 3.00798 & 4.91300 & 0.40323 & 3.000 & 0.40323 & & & & & & & \\
\hline 1.24967 & 8.35210 & 0.68544 & 3.000 & 0.04113 & 16.667 & 4 & 6 & 3 & ! & 8 & 2 \\
\hline 0.00000 & 8.35210 & 0.68549 & 3.000 & 0.03884 & 17.647 & 4 & 0 & 0 & 2 & 8 & 2 \\
\hline 3.99361 & 8.35210 & 0.68549 & 3.000 & 0.02742 & 25.000 & 4 & 7 & 1 & 3 & 9 & 1 \\
\hline 0.00000 & 8.35210 & 0.68549 & 3.000 & 0.03834 & 17.647 & 4 & 0 & 0 & 4 & 9 & 1 \\
\hline 0.04417 & 8.35210 & 0.68549 & 3.000 & 0.03884 & 17.647 & 4 & 7 & 1 & 5 & 8 & 1 \\
\hline 0.00000 & 0.35210 & 0.68549 & 3.000 & 0.03884 & 17.647 & 4 & 0 & 0 & 6 & 8 & 1 \\
\hline 4.64053 & 8.35210 & 0.68549 & 3.000 & 0.68549 & & & & & & & \\
\hline 2.77404 & 14.19857 & 1.16533 & 3.000 & 0.06992 & 16.667 & 4 & 6 & 3 & I & 8 & 2 \\
\hline 0.00003 & 14.19857 & 1.16533 & 3.000 & 3.06604 & 17.647 & 4 & 0 & 0 & 2 & 8 & 1 \\
\hline 5. 30085 & 14.19857 & 1.16533 & 3.000 & 0.04661 & 25.000 & 4 & 7 & 1 & 3 & 9 & 1 \\
\hline 0.00000 & 14.19857 & 1.16533 & 3.000 & 0.06604 & 17.641 & 4 & 0 & 0 & 4 & 9 & 1. \\
\hline 0.26780 & 14.19857 & 1.16533 & 3.000 & 3.06604 & 17.647 & 4 & 7 & 1 & 5 & 8 & 1 \\
\hline 0.00000 & 14.19857 & 1.16533 & 3.000 & 0.06604 & 17.647 & 4 & 0 & 0 & 6 & $\mathbf{B}$ & 1 \\
\hline 7.31177 & 14.19857 & 1.16533 & 3.000 & 1.16533 & & & & & & & \\
\hline 5.22260 & 24.13757 & 1.98106 & 3.000 & 0.11886 & 16.667 & 4 & 6 & 3 & I & 8 & 2 \\
\hline 0.00000 & 24.13757 & 1.98106 & 3.000 & 0.11226 & 17.647 & 4 & 0 & 0 & 2 & 8 & 1 \\
\hline 7.87578 & 24.13757 & 1.98106 & 3.000 & 0.07924 & 25.000 & 4 & 7 & 1 & 3 & 9 & 1 \\
\hline 0.20000 & 24.13757 & 1.98106 & 3.000 & 3.11226 & 17.647 & 4 & 0 & 0 & 4 & 9 & 1 \\
\hline 0.69556 & 24.13757 & 1.98106 & 3.000 & 0.11226 & 17.647 & 4 & 7 & 1 & 5 & 8 & 1 \\
\hline 0.00000 & 24.13757 & 1.98106 & 3.000 & 3.11226 & 17.641 & 4 & 0 & 0 & 6 & 8 & 1 \\
\hline 10.83486 & 24.13757 & 1.98106 & 3.000 & 1.98106 & & & & & & & \\
\hline 7.55978 & 41.03387 & 3.36780 & 3.000 & 0.20207 & 16.667 & 4 & 6 & 3 & 1 & 8 & 2 \\
\hline 0.00011 & 41.03387 & 3.36780 & 3.000 & 0.19084 & 17.647 & 4 & 0 & 0 & 2 & 8 & 1 \\
\hline 9.01608 & 41.03387 & 3.36780 & 3.000 & $0:-13471$ & 25.000 & 4 & 7 & 2 & 3 & 9 & 1 \\
\hline 0.00011 & 41.03387 & 3.36780 & 3.000 & 0.19084 & 17.647 & 4 & 0 & 0 & 4 & 9 & 1 \\
\hline 1.22761 & 41.03387 & 3.36780 & 3.000 & 0.19084 & 17.647 & 4 & 7 & 1 & 5 & 8 & 1 \\
\hline 0.00011 & 41.03387 & 3.36780 & 3.000 & 0.19084 & 17.647 & 4 & 0 & 0 & 6 & 8 & 1 \\
\hline
\end{tabular}

\begin{tabular}{|c|c|c|c|c|c|c|c|c|c|c|c|}
\hline 13.40088 & 41.03387 & 3.36780 & 3.000 & 3.36780 & & & & & & & \\
\hline 7.25440 & 69.75757 & 5.72526 & 3.000 & 0.34352 & 16.667 & 4 & 6 & 3 & 1 & 8 & 2 \\
\hline $0.0105 \theta$ & 69.75757 & 5.72526 & 3.000 & 0.32443 & 17.647 & 4 & 6 & 0 & 2 & 8 & 0 \\
\hline 7.58001 & 69.75757 & 5.72526 & 3.000 & 0.22901 & 25.000 & 4 & 6 & 2 & 3 & 9 & 1 \\
\hline 0.01066 & 69.75757 & 5.72526 & 3.000 & 0.32443 & 17.647 & 4 & 6 & 0 & 4 & 9 & 0 \\
\hline 1.56164 & 69.75757 & 5.72526 & 3.000 & 0.32443 & 17.647 & 4 & 6 & l & 5 & 8 & 1 \\
\hline 0.01068 & 69.75757 & 5.72526 & $3.0,00$ & 0.32443 & 17.647 & 4 & 4 & 0 & 6 & 8 & 0 \\
\hline 11.94868 & 69.75757 & 5.72526 & 3.000 & 5.72526 & & & & & & & \\
\hline 4.12965 & 118.58787 & 9.73295 & 3.000 & 0.58398 & 16.667 & 4 & 6 & 3 & 1 & 8 & 2 \\
\hline 0.13184 & $\mid 1 H . D B I H\}$ & Y. $13<43$ & 3.0000 & 0.35133 & 17.047 & 4 & 6 & 0 & 2 & 0 & 0 \\
\hline 4.06738 & 118.58787 & 9.73295 & 3.000 & 0.38932 & 25.000 & 4 & 6 & 2 & 3 & 9 & 1 \\
\hline 0.13367 & 118.58787 & 9.73295 & 3.000 & 0.55153 & 17.647 & 4 & 6 & 0 & 4 & 9 & 0 \\
\hline 1.60361 & 128.58787 & 9.73295 & 3.000 & .0 .55153 & 17.647 & 4 & 6 & 1 & 5 & B & 1 \\
\hline 0.13415 & 118.58787 & 9.73245 & 3.000 & 0.55153 & 17.647 & 4 & 5 & 0 & 6 & 8 & 0 \\
\hline 7.06135 & 118.58787 & 9.73295 & 3.000 & $\nexists 7.73295$ & & & & & & & \\
\hline
\end{tabular}

7040 TIME FOR THIS CASE 2.7694 E-OI MINUTES 


\section{TABIE VI}

Part of Print-Out of $\bar{\sigma}_{\mathcal{X}}\left(\mathrm{E}_{0}, \mathrm{E}_{i}\right)$ Calculation For Water Using the Three Aggregate Model (Five Groups in the Range $0 \leqq \mathrm{E} \leqq 0.0125 \mathrm{eV}$ )

TOTALS EO $=0.00125 \quad \mathrm{PO}=0.23029435 \mathrm{E} 03 \quad \mathrm{P} 1=0.56668922 \mathrm{E} \quad 02 \quad \mathrm{P} 2=0.40537650 \mathrm{E} 02$ $\mathrm{P} 3=0.29553441 \mathrm{E} 02$

PO for $\mathrm{EO}=0.00125$ to $\mathrm{E}$
$0.98317589 \mathrm{E} 05$
$0.10130321 E 05$
$0 .: 30702490 \mathrm{E} 04$
$0.25992240 \mathrm{E} 04$
$0.34396741 \mathrm{E} 04$

$\mathrm{PI}$ for $\mathrm{EO}=0.00125$ to $\mathrm{E}$

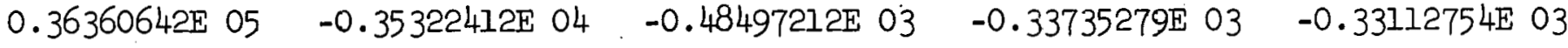

$\mathrm{P} 2$ for $\mathrm{EO}=0.00125$ to $\mathrm{E}$

$\begin{array}{lllllllll}0.22093472 \mathrm{E} \text { o5 } & -0.37979645 \mathrm{E} & 03 & 0.85394693 \mathrm{E} & 02 & -0.3779=891 \mathrm{E} & 02 & -0.30466371 \mathrm{E} & 02\end{array}$

$\mathrm{P} 3$ for $\mathrm{EO}=0.00125$ to $\mathrm{E}$

$0.15837281 \mathrm{E}$ 05 $\quad 0.35934669 \mathrm{E} 02$

$-0.37040476 \mathrm{E} 02$

$-0.32300030902$

$-0.30904849 \mathrm{E} 02$

PO $\mathrm{EO}(\mathrm{I})$ to $\mathrm{E}=0.00125$

$0.98317589 \mathrm{E}$ of $\quad 0.37282639 \mathrm{E} 04$.

$0.74853648 \mathrm{E} 03$

0.4997579603

$0.56792830 \mathrm{E} 03$

$\mathrm{Pl} \mathrm{EO}(\mathrm{I})$ to $\mathrm{E}=0.00125$

$0.36360642 \mathrm{E}$ o5 $\quad-0.12999713 \mathrm{E} 04$

$-0.11823774 \mathrm{E} \mathrm{O3}$

$-0.64853491 \mathrm{IE} 02$

$-0.54672825 \mathrm{E} 02$

$\mathrm{P} 2 \mathrm{EO}(\mathrm{I})$ to $\mathrm{E}=0.00125$

$0.22093472 \mathrm{E}$ 05 $-0.13977655 \mathrm{E} 03$

$0.20819498 \mathrm{E}$ o2

$-0.72663218 \Xi 01$

$-0.50303354 \mathrm{E} 01$

P3 $\mathrm{EO}(\mathrm{I})$ to $\mathrm{E}=0.00125$

$0.15837281 \mathrm{E}$ 05 $0.13225042 \mathrm{E}$ O2

$-0.90305858 \mathrm{E} \mathrm{OI}$

$-0.62103909 \mathrm{I} \mathrm{OI}$

$-0.51027330 \mathrm{E} \cdot 01$ 
order for $\ell=0,1,2,3$ it gives $\Sigma_{i} \bar{\phi}\left(E_{0}, E_{i}\right) \Delta E_{i}$. Here $i$ denotes the group. The $\bar{\sigma}_{l}\left(E_{0}, E_{i}\right)$ is computed for $E_{i}$ in the center of the group and $\Delta E_{i}$ is the group width. For fine enough intervals, the $l=0$ total is just the total scattering cross section $\bar{\sigma}\left(E_{0}\right)$.

The values will be questionable unless the group widths are fine enough so that the variation of $\bar{\sigma}_{l}\left(E_{0}, E\right)$ across the group is nearly linear.

Under PO FOR $E_{O}=0.00125$ TO $E$ are listed the value of $\bar{\sigma}_{l}\left(E_{0}, E_{i}\right)$. for each group i. Similarly PI for EO TO E down to P3 for EO to E give the corresponding values of $\bar{\sigma}_{l}\left(\mathrm{E}_{0}, \mathrm{E}_{i}\right)$. Finally, the values for $\bar{\sigma}_{l}\left(E_{i}, E_{0}\right)$ are listed under PO EO(I) to $E=0.00125$, etc. 
REFTERENCES

1. G. Placzek, Phys. Rev. 56, 377-388 (1952).

G. C. Wick, Phys. Rev. 24, 1228-1242 (1954).

M. S. Nelkin, "Proceedings of the Symposium on Inelastic Scattering of Neutrons in Solids and Liquids", Vienna, October 11-14, 1960, pp 3-25.

2. I. Van Hove, Phys. Rev. 95, 249-262 (1954).

3. A. C. Zemach and R. J. Giauber, Phys. Rev. 101, 118-129 (1956).

4. P. Schofield, Phys. Rev. Letters 4, 239-240 (1960).

P. Egelstaff and P. Schofield, Nuc. Sci. and Engr. 12, 260-270 (1962).

5. H. I. McMurry, Nuc. Sci. and Engr. 15, 429-437 (1963).

6. H. L. McMurry, "Calculation of Differential Scattering Cross Sections in the Short Collision Time Approximation", DDO-16749 (April, 1962). This repart uses time in units of $x / E_{0}$ instead of 不/ kT.

7. M. S. Nelkin, Phys. Rev. 119 741-746 (1.960)

8. B. C. Haywood, A.E.R.E. - R4489 (1964)

9. See, for example, I. Pelah and J. Imry IA-875 (1964)

J. D. Bermal and R. H. Fowler, J. Chem. Phys. I 515 (1933).

L. Pauling, "The Nature of the Chemical Bond", Chap X. Cornell University Press (1939). 
REFERENCES (Continued)

10. Neutron Cross Sections, BNL 325, July 1958. 


\section{APPENDIX}

The $S\left(\kappa^{2}, \beta\right)$ Function as a Fourier Transform

The function $S_{v}\left(\kappa^{2}, \beta\right)$ can be written as a Fourier transform similar to that for $\sigma_{v}\left(E_{0}, E, \mu\right)$. The appropriate expression is obtained by putting $t=t-i / 2$ in Eq. (18), or its classical or pure quantum counterparts (4). Conditions under which the S.C.T. and classical equations for $s_{v}\left(\kappa^{2}, \beta\right)$ are equivalent can be discussed in terme of the Fourier time traneform.

When $t-1 / 2$ is substituted for $t$ in Eq. (18) and the factor $\left(E / E_{D}\right)^{\frac{1}{2}} \exp (-\beta / 2)$ is extracted the remaining factor is the following expression for $\overline{\mathrm{S}}_{\psi}\left(\kappa^{2}, \beta\right)$

$$
\begin{aligned}
& \bar{S}_{\nu}\left(k^{2}, \beta\right)=\frac{A_{v}^{2}+c_{\nu}^{2}}{2 \pi k T} \exp \frac{-\left(\bar{\alpha}_{v}+2 \bar{\alpha}_{v \lambda}\right)}{4} \int_{-\infty}^{\infty} e^{-i t\left(\beta+\bar{\alpha}_{v \lambda}\right)} e^{-\bar{\alpha}_{v} t^{2}} \\
& \left.x<0_{V \lambda \lambda} \pi_{T}<0_{V \lambda}^{1}\right\rangle_{T} d t
\end{aligned}
$$

If this time shift is inserted into the expansion for $\left\langle\overline{0}_{V \lambda}^{\prime} \text { (it) }\right\rangle_{T}$ there results

$$
\begin{aligned}
& \left.\overline{0}_{\nu \lambda}\right\rangle_{T}=\sum_{l=0}^{\infty}-\frac{(i t)^{l}}{\bar{a}_{l}} \frac{-}{l !} \\
& \bar{a}_{\ell}=\sum_{n=l}^{\infty} \frac{\left\langle\overline{0}^{-(n)}\right\rangle_{T}}{2^{n-l}(n-l) !}
\end{aligned}
$$

The expression for $\left\langle\bar{O}_{V_{T}^{\prime}}^{\prime}\right\rangle_{T}$ is obtained by inserting the time shift into Eq. (20) of Ref. (ㄷ). The result is

$$
\left\langle\overline{0}_{\nu T T}^{\prime}\right\rangle_{T}={\overline{x_{\nu T}}}_{\nu} I_{0}\left(\bar{g}_{\nu \tau}\right)\left\{I+2 \sum_{n_{T}=1}^{\infty} \frac{I_{n T}\left(\bar{g}_{\nu \tau}\right)}{I_{0}\left(\bar{g}_{\nu \tau}\right)} \cosh \left(i \operatorname{tin} \beta_{\tau}\right)\right\}
$$


When the $\lambda$ mode is treated classically the result analogous to Eq. $(A-1)$ is

$\bar{s}_{\nu}\left(\kappa^{2}, \beta\right)=\frac{A_{v}^{2}+C_{v}^{2}}{2 \pi k T} \exp \frac{-\left(\bar{\alpha}_{v}+\bar{\alpha}_{v \lambda}\right)}{4} \int_{-\infty}^{\infty} e^{-i t \beta_{e}} e^{-\left[\bar{\alpha}_{\nu}+\bar{\alpha}_{\nu \lambda}\right] t^{2}}$

$$
\times \pi_{T}<\overline{0}_{V T}^{\prime}>_{T} d t
$$

Equations (A-I) and $A-5)$ will be the same provided.

$$
e^{-\bar{\alpha}_{v \lambda} / 4} e^{-i t \bar{\alpha}_{\nu \lambda}} e^{-\bar{\alpha}} v t^{t^{2}}\left\{\sum_{l l=0}^{\infty} \bar{a}_{l} \frac{(i t)^{l}}{l !}\right\}=1
$$

This can be achieved only when $\beta_{\lambda}$ is sufficiently small, as has been discussed in connection with Eq. (18).

Even when conditions insure it is valid to assume $\left\langle\overline{0}_{V \lambda}\right\rangle_{T} \simeq 1$

Eq. (A-I) will not give a result which is even in $\beta$. The result is*

$\bar{s}_{\nu}\left(k^{2}, \beta\right)=C_{v}\left(1 / \alpha_{v}\right)^{\frac{1}{2}} \exp \frac{-\left(\bar{\alpha}_{v}+2 \bar{\alpha}_{v \lambda}\right)}{4} \exp \frac{-\left(\beta+\bar{\alpha}_{v \lambda}\right)^{2}}{4 \bar{\alpha}_{v}} \bar{\psi}_{v S}$

$\bar{c}_{v}=\frac{A_{v}^{2}+C_{v}^{2}}{2 k T \sqrt{\pi}} \bar{x}_{v_{g}}^{O}>_{T} \pi_{T} I_{0}\left(\bar{g}_{\nu T}\right)$

$\left.\bar{x}_{\nu q^{0}}^{0}\right\rangle_{T}=\exp -\left(\Sigma_{T} \bar{g}_{\nu T} \cosh \left(\beta_{\tau} / 2\right)\right)$

$\bar{\Psi}_{v S}=1+2 \sum_{n_{1}, n_{2} \cdots n_{T}} \frac{I_{n \tau}\left(\bar{g}_{\nu \tau}\right)}{I_{0}\left(\bar{g}_{v \tau}\right)}\left[\sum_{i} e^{\frac{-x_{1}^{2}}{4 \alpha_{v}}} \cosh \frac{\left(\beta+\bar{\alpha}_{\nu \lambda}\right) x_{i}}{2 \bar{\alpha}_{v}}\right]$

In Eqs. (A-7) - (A-9) subscripts $T$ denote modes which are treated quantum mechanically, and $\lambda$ pertains to the mode treated in S.C.T.

* Reference () has an error in that $\beta$ is used in place of

$\bar{\beta}+\sum_{\lambda} \bar{\alpha}_{V \lambda}$ in the equivalent to Eq. $(A-9)$. 
approximation. In Eq. (A-9) the $x_{i}$ are as defined in connection with Eq. (24). The classical equivalent to Eq. (A-7) is

$\bar{s}\left(\kappa^{2}, \beta\right)=\bar{c}_{v}\left[\frac{1}{\bar{\alpha}_{v}+\bar{\alpha}_{v \lambda}}\right]^{\frac{1}{2}} \exp \frac{-\left[\bar{\alpha}_{v}+\bar{\alpha}_{v \lambda}\right]}{4} \exp \left[\frac{-\beta^{2}}{4\left(\bar{\alpha}_{v}+\bar{\alpha}_{v \lambda}\right)}\right] \bar{\psi}_{v c}(A-10)$

The $\bar{\psi}_{v_{C}}$ in Eq. $(A-10)$ is obtained from $\bar{\psi}_{V_{S}}$ by replacing $\bar{\alpha}_{v}$ by $\bar{\alpha}_{\nu}+\bar{\alpha}_{\nu \lambda}$, and $\beta+\bar{\alpha}_{v \lambda}$ by $\beta$.

Equations $(A-7)$ and $(A-10)$ will be alike if $\bar{\alpha}_{\nu \lambda} \ll \bar{\alpha}_{\nu}$. This requirement is met if $\beta_{\lambda}>1$ and the condition (23b) is fulfilied. If (230) is fulfilled when $\bar{\alpha}_{V \lambda} \sim \bar{\alpha}_{\nu}$ and $\beta_{\lambda}<1$ it is likely that the classical result is valid because $\beta_{\lambda} \rightarrow 0$.

If (23b) is not fulfilled but (23c) is, several possibilities exist. It may still be true that $\bar{\alpha}_{v \lambda} \ll \bar{\alpha}_{\nu}$ and this could result in (23c) being satisfied under conditions which insure the validity of the classical equation.

If in this situation, $\bar{\alpha}_{V \lambda} \geq \bar{\alpha}_{v}$ it may happen that $\bar{\alpha}_{V \lambda} \ll \beta$. It is then likely that the factor $\exp \left(-\beta^{2} / 4 \alpha{ }_{v}\right)$ will make $\bar{s}_{v}\left(k^{2} / \beta\right)$ small so it really does not matter if the classical equation is used. Similarly, if $\bar{\alpha}_{\nu \lambda} \geq \bar{\alpha}_{v}$ and $|\beta| \leq \bar{\alpha}_{v \lambda}$ (23) will be satisfied only for large $\bar{\alpha}_{V \lambda}$. This implies $\bar{s}_{v}\left(\kappa^{2}, \beta\right)$ will be small because of the factor $\exp \left[-\bar{\alpha}_{v \lambda} / 4\right]$ so that little difference results from using the classical expression.

It appears likely that the criteria (23 a-c) will insure that a classical calculation is adequate. 


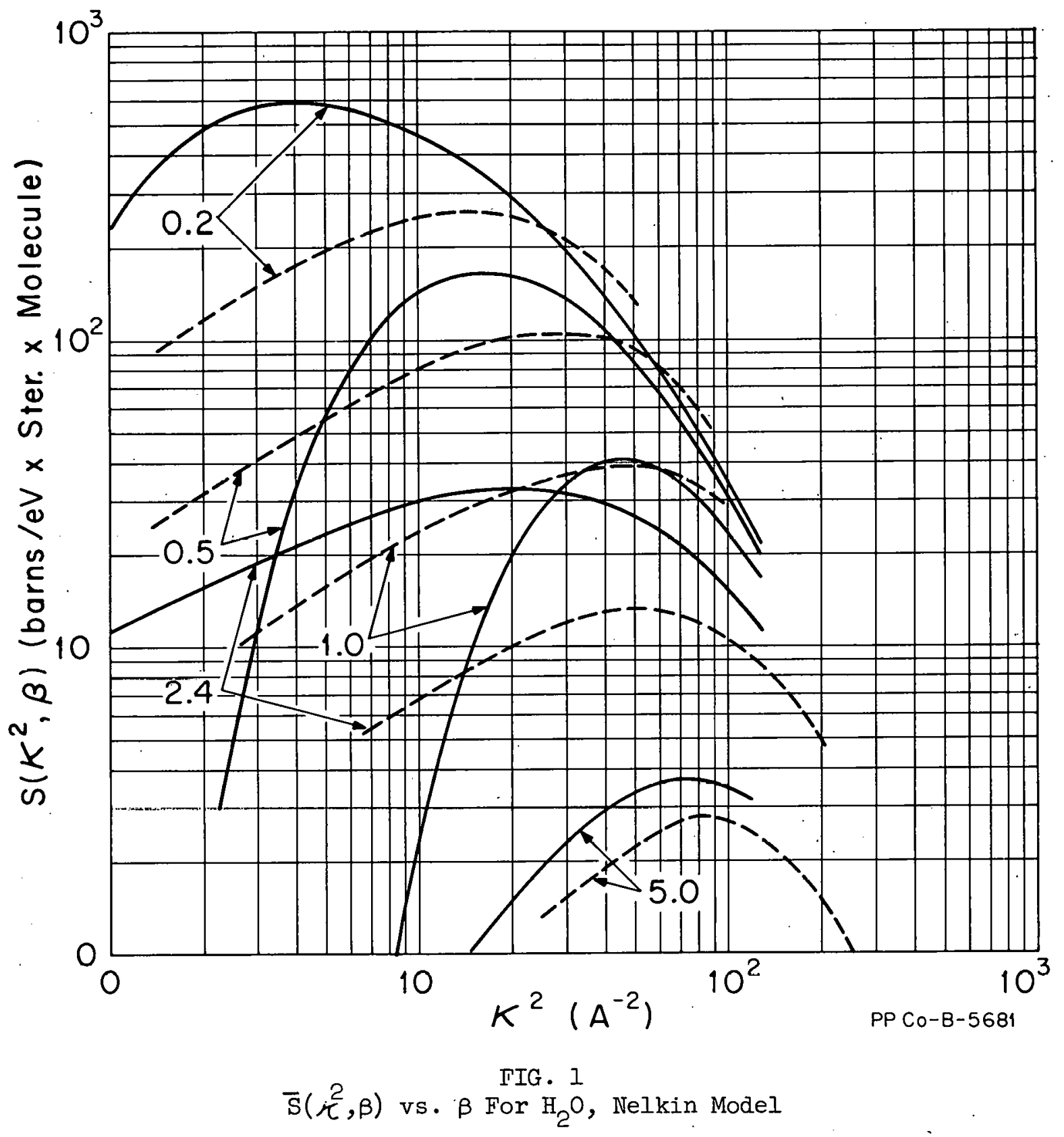




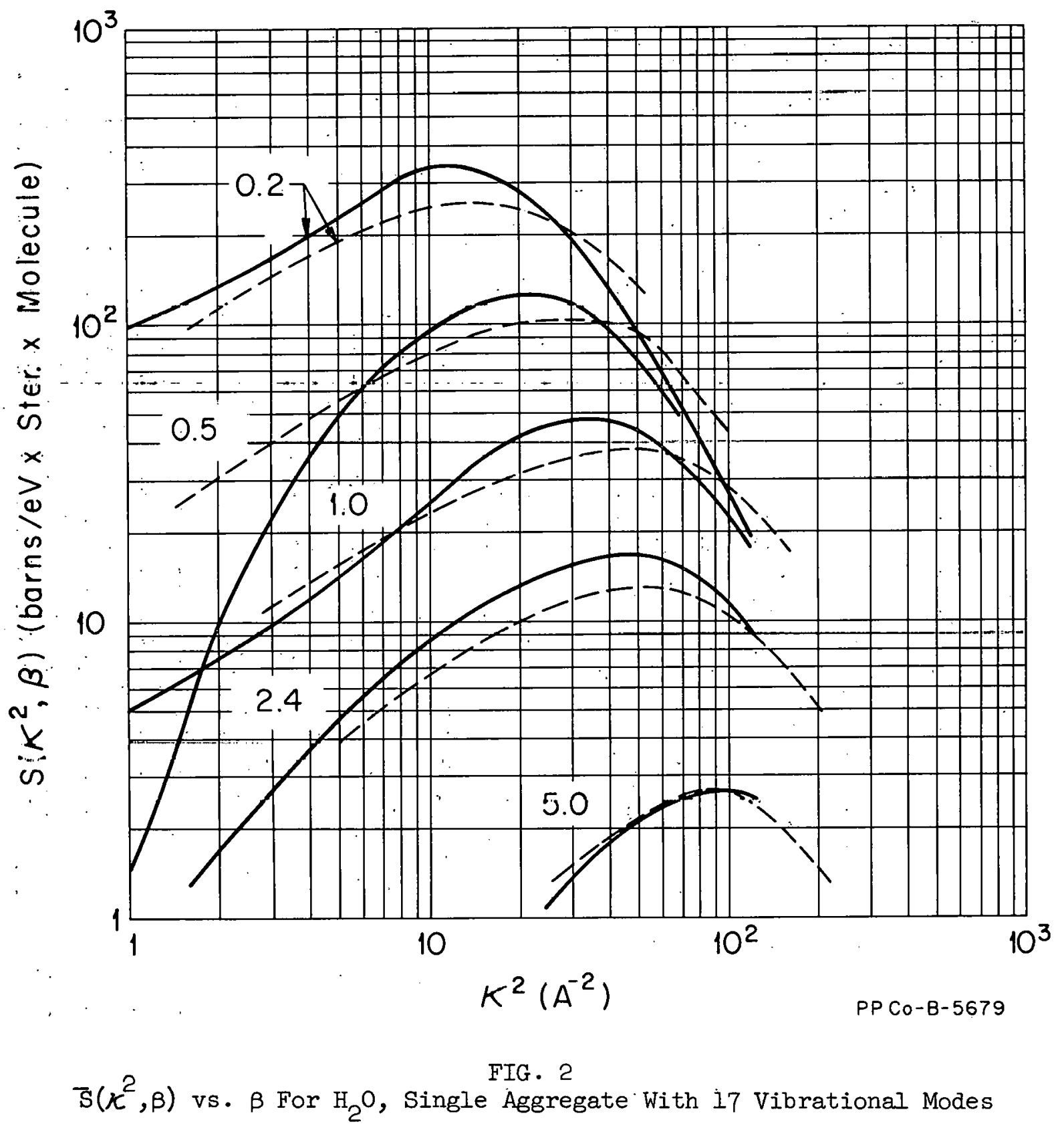




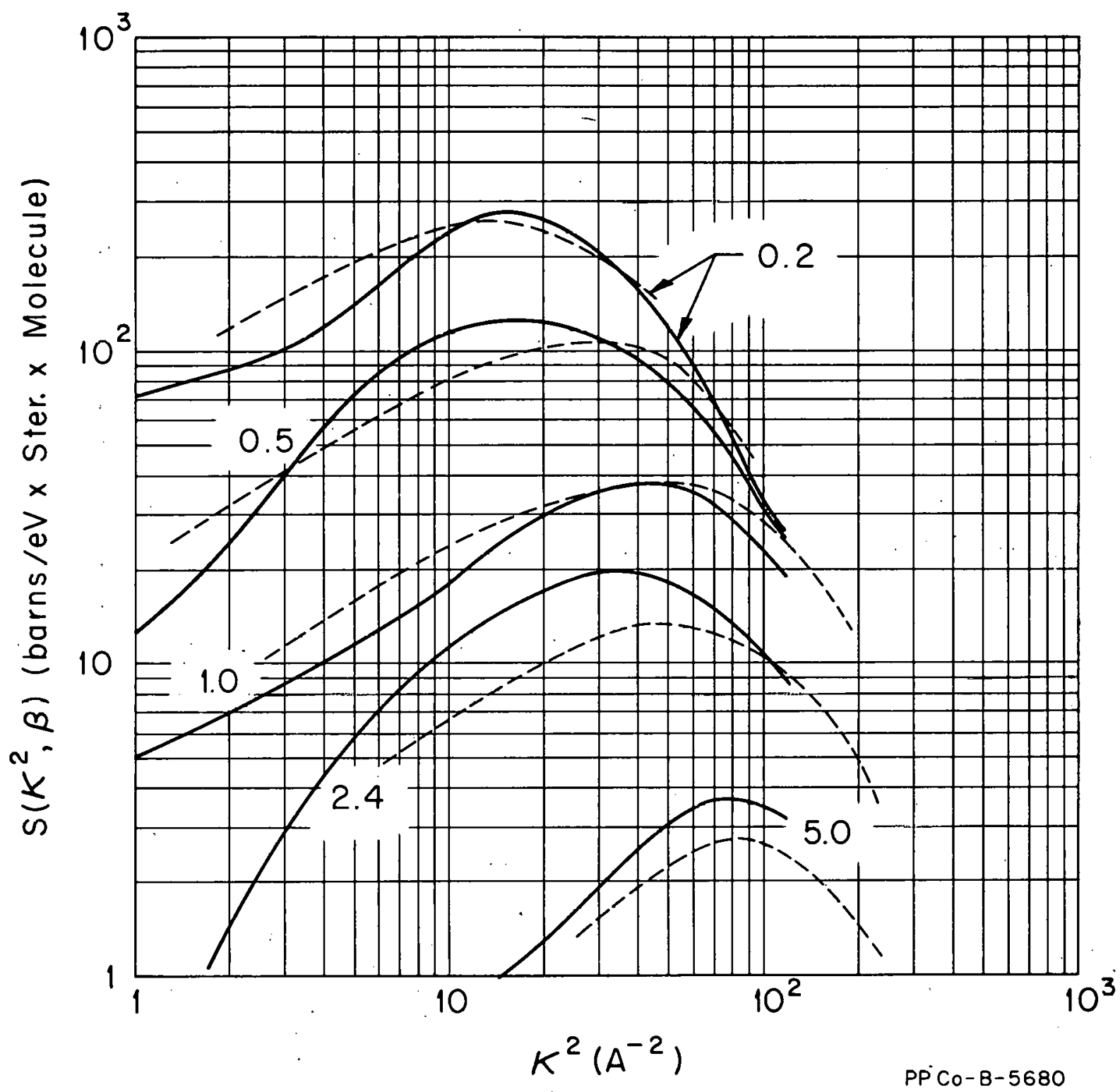

FIG. 3

$\overline{\mathrm{S}}\left(\kappa^{2}, \beta\right)$ vs: $\beta$ For $\mathrm{H}_{2} \mathrm{O}$, Three Aggregate Model 


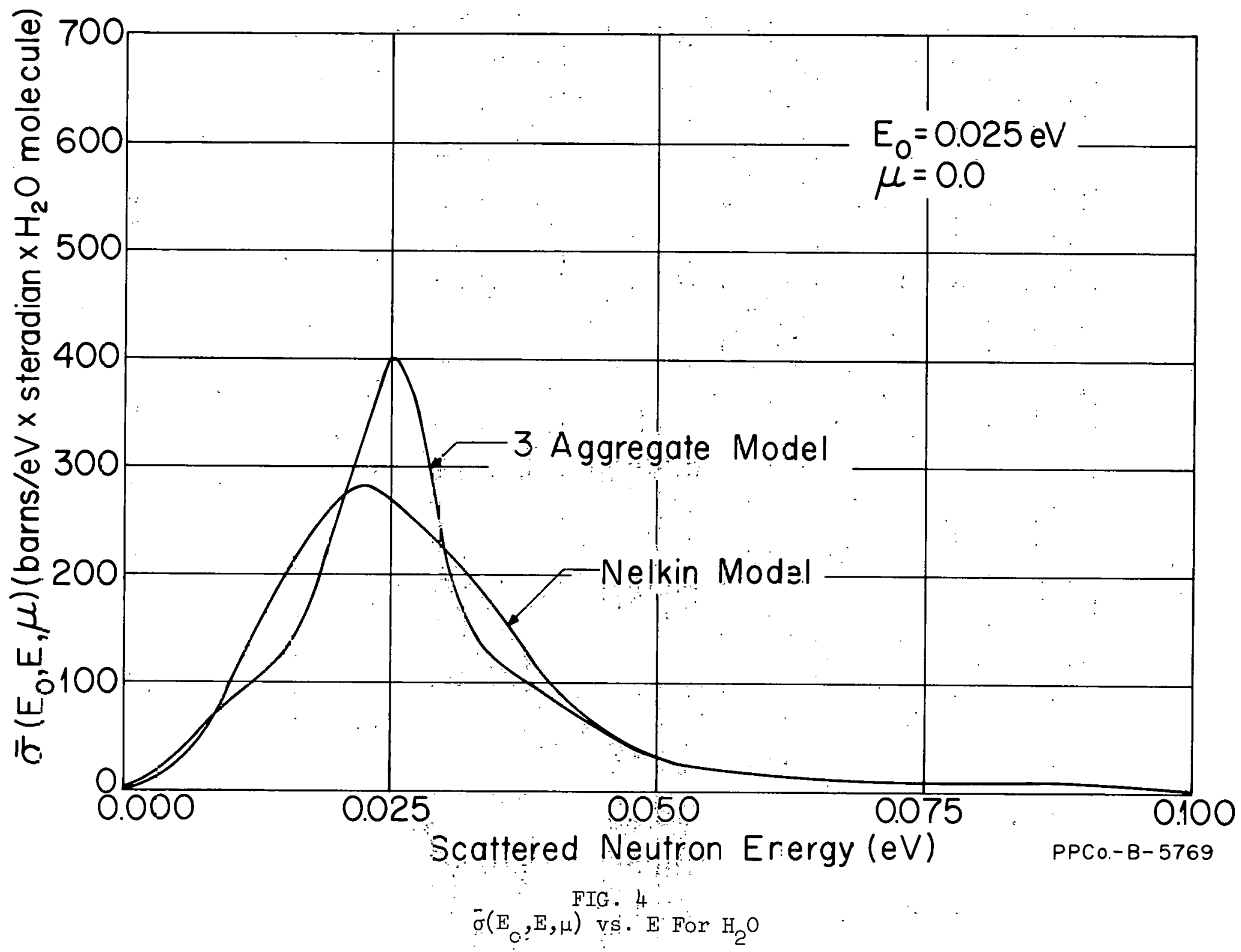




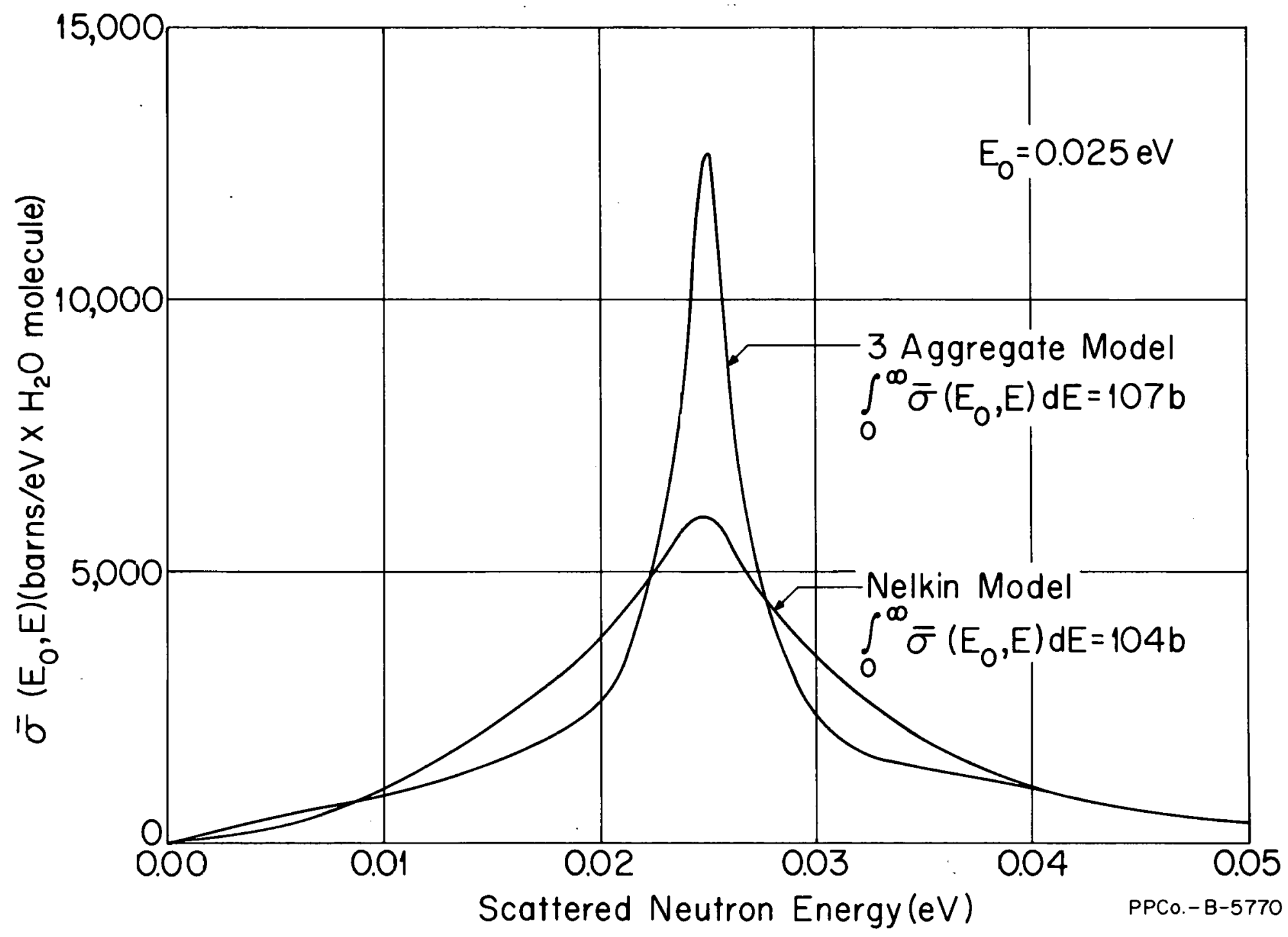

$$
\bar{\sigma}\left(\mathrm{E}_{\mathrm{O}}, \mathrm{E}\right) \stackrel{\text { FIG. }}{5} \text { vs. } \mathrm{E} \text { For } \mathrm{H}_{2} \mathrm{O}
$$




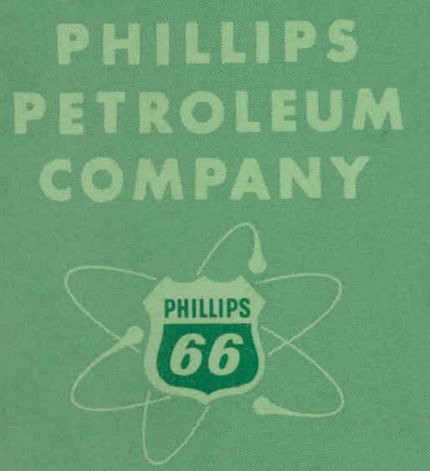

ATOMIC ENERGY DIVISION 